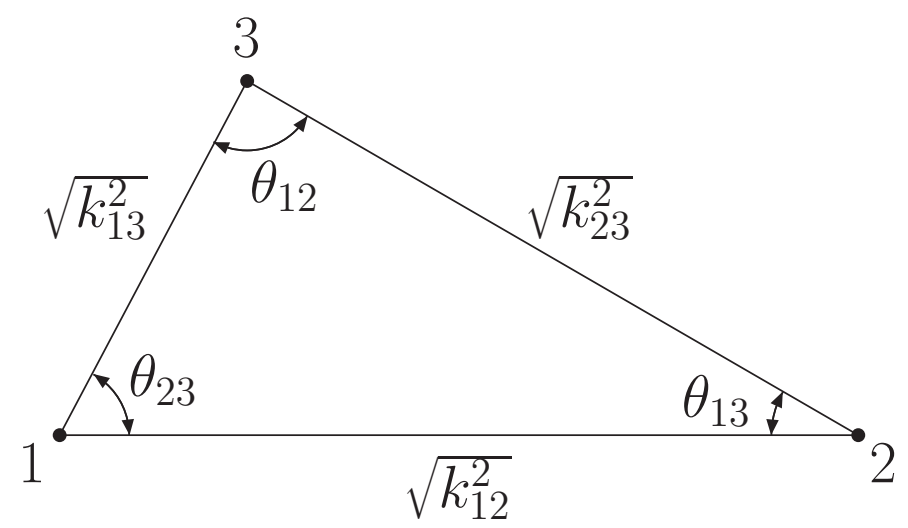

Fig. 1. The triangle associated with the massless three-point function

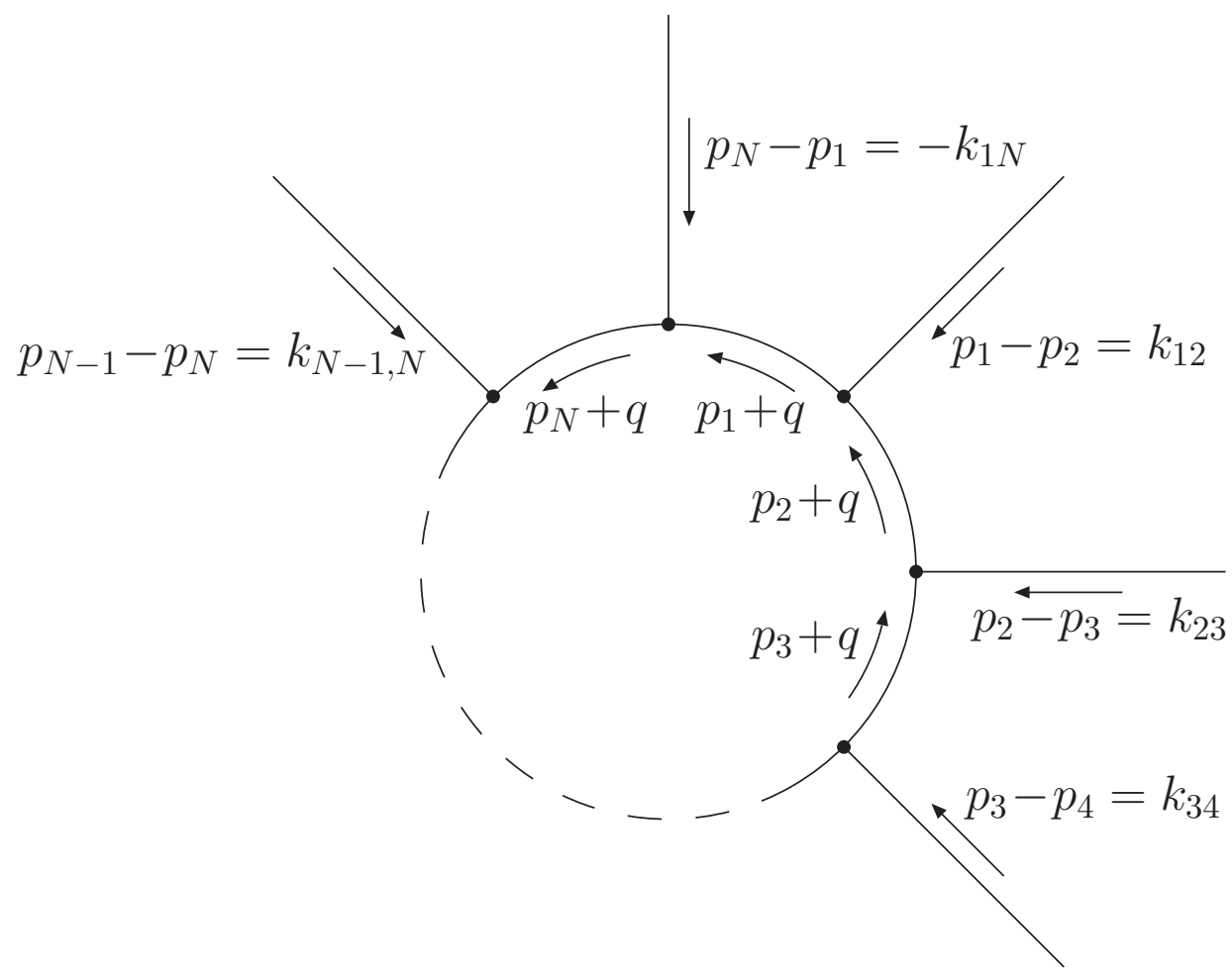

Fig. 2. The one-loop $N$-point diagram 


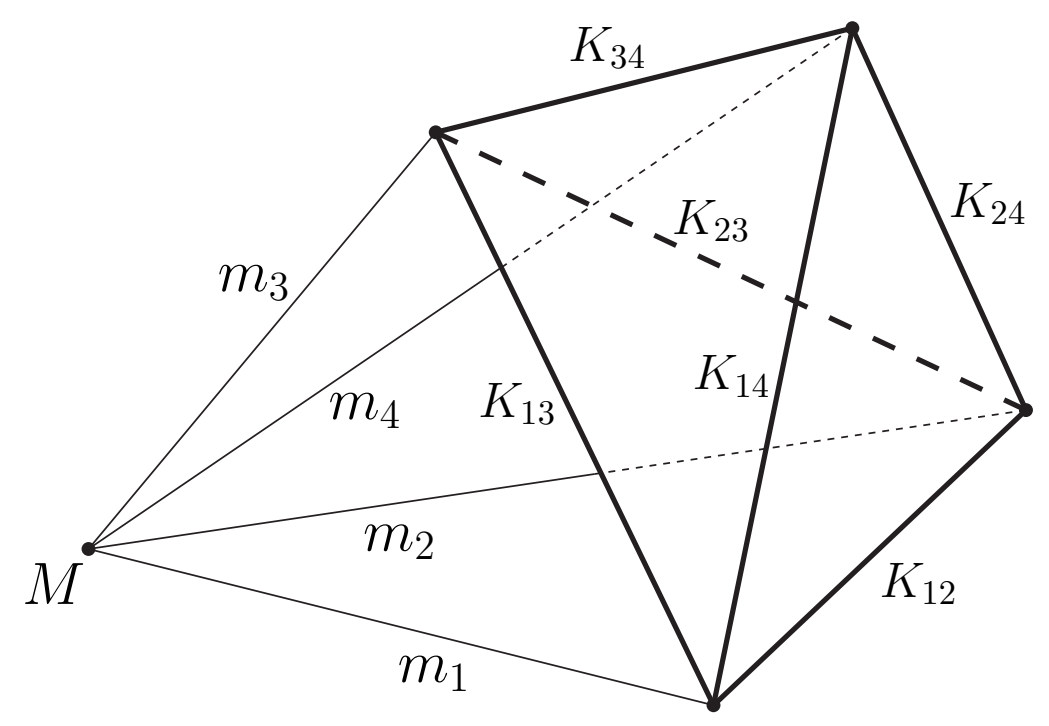

Fig. 3. The basic simplex for $N=4$

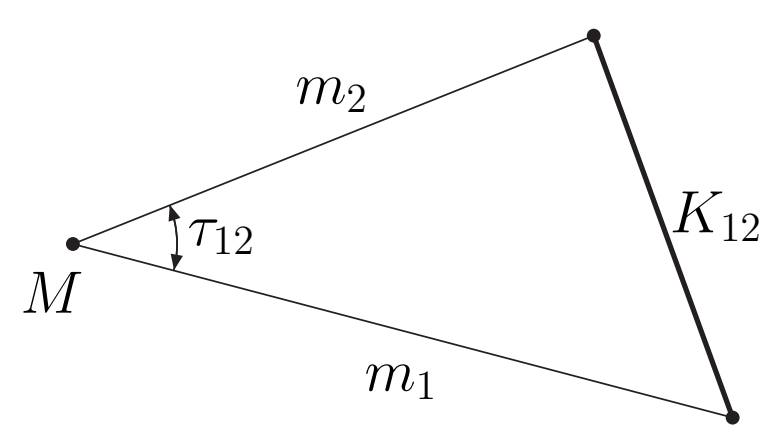

(a)

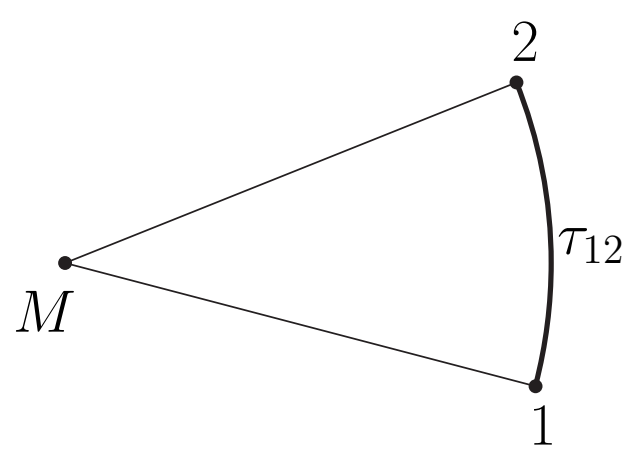

(b)

Fig. 4. Two-point case: (a) the basic triangle and (b) the arc $\tau_{12}$

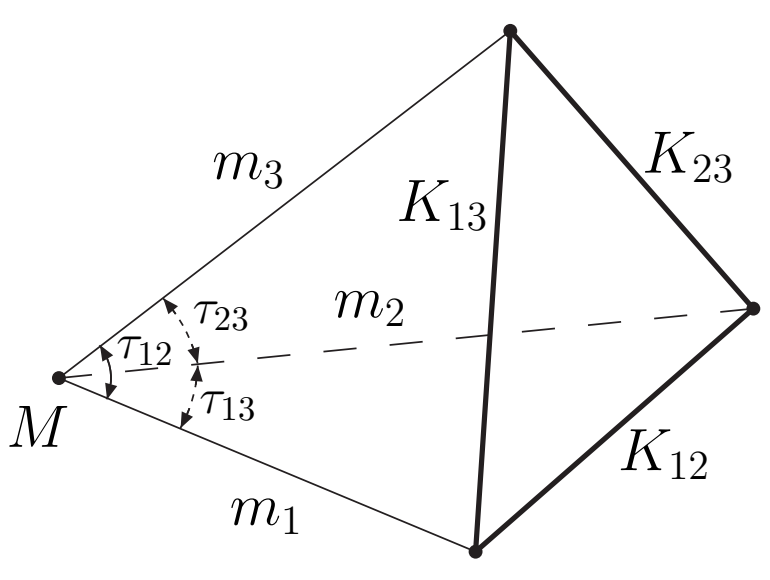

(a)

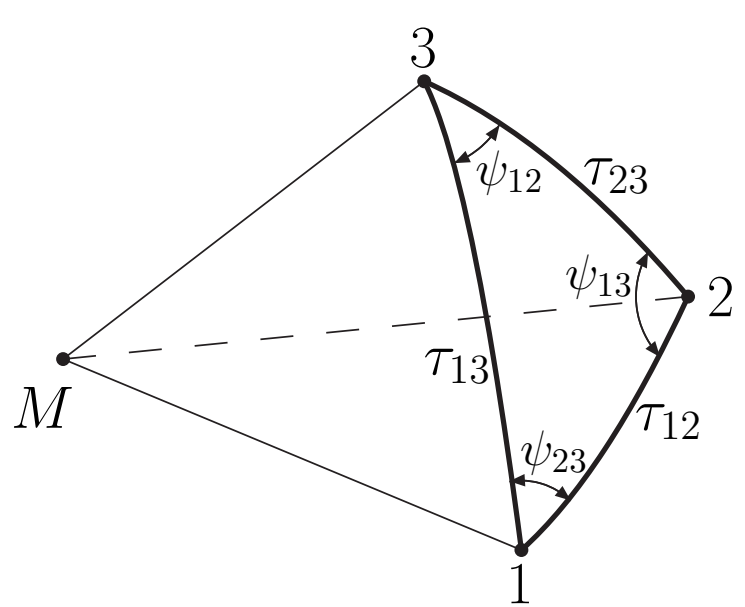

(b)

Fig. 5. Three-point case: (a) the basic tetrahedron and (b) the solid angle 


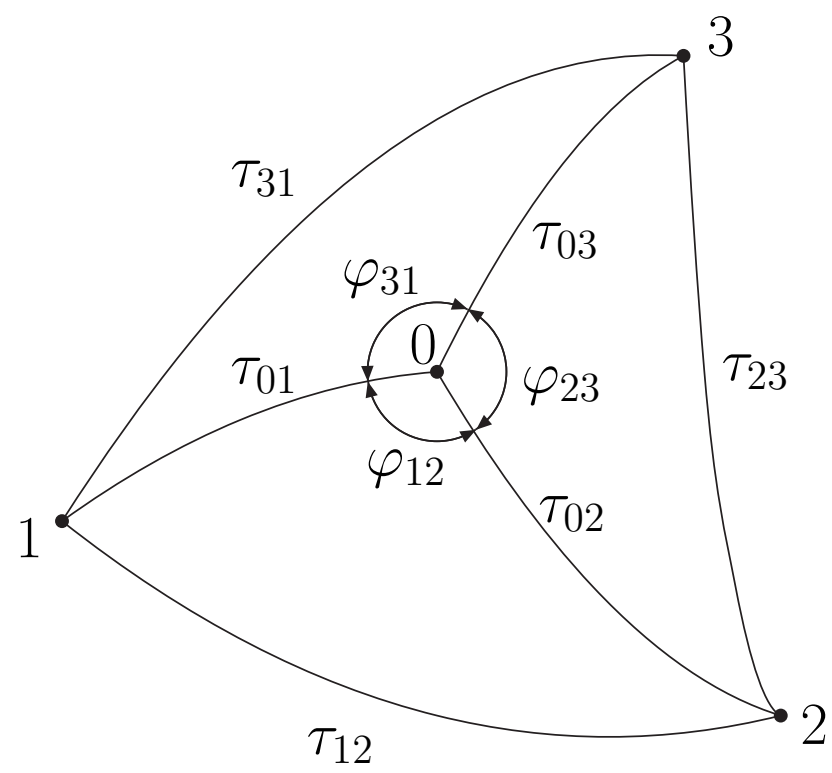

Fig. 6. The spherical triangle 123

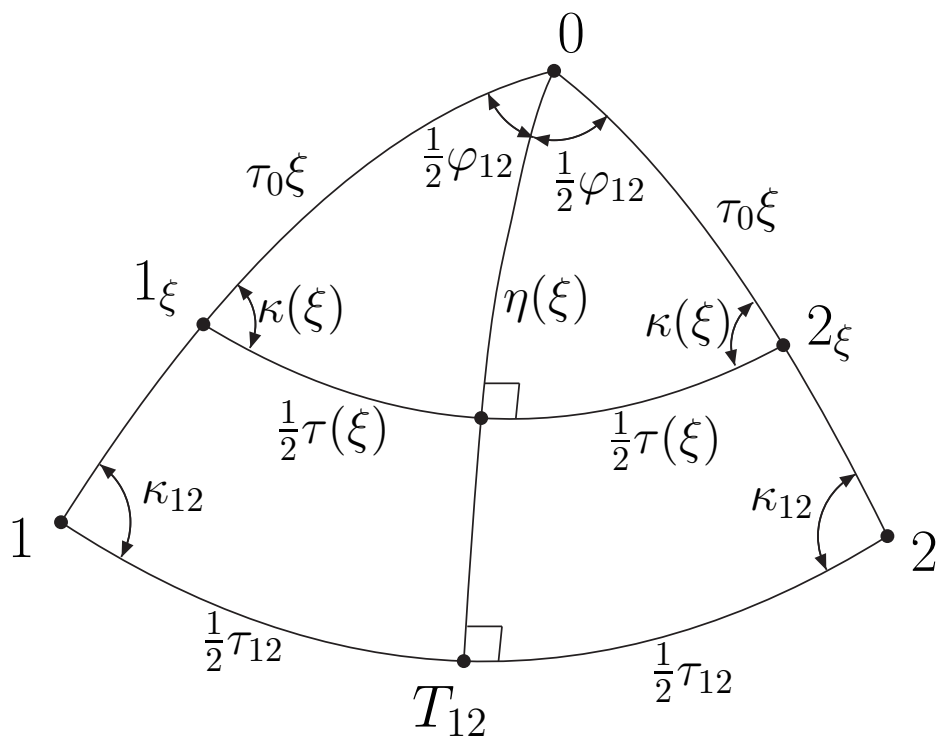

Fig. 7. The isosceles spherical triangle 012 


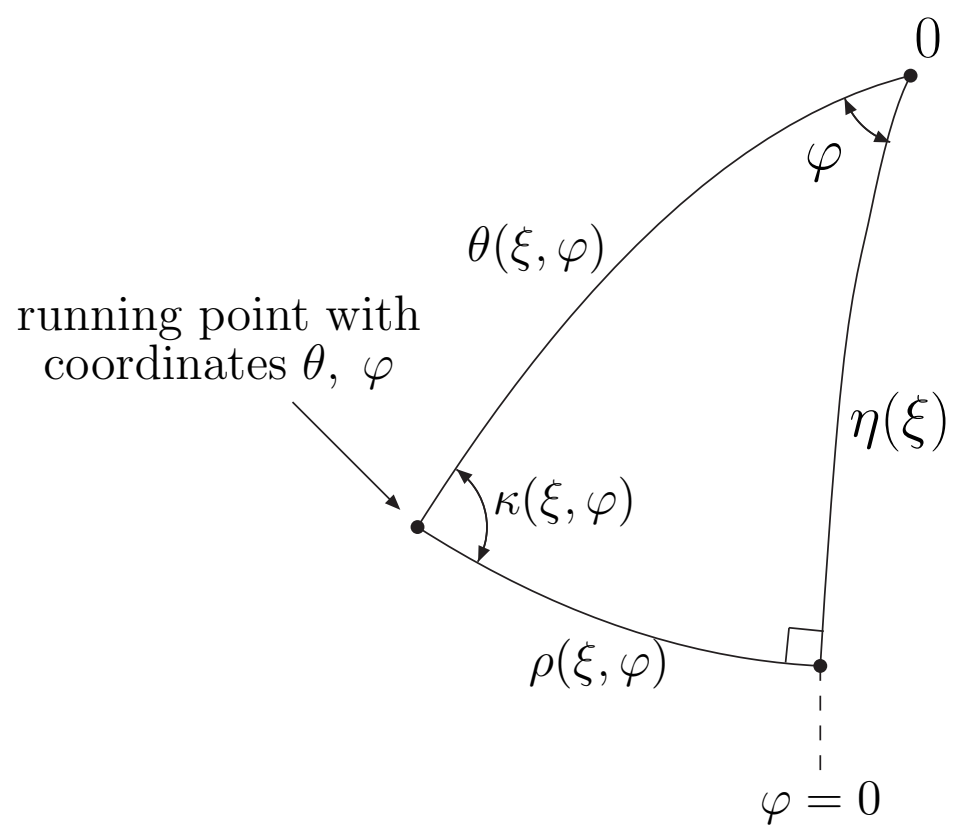

Fig. 8. Integration variables on the sphere

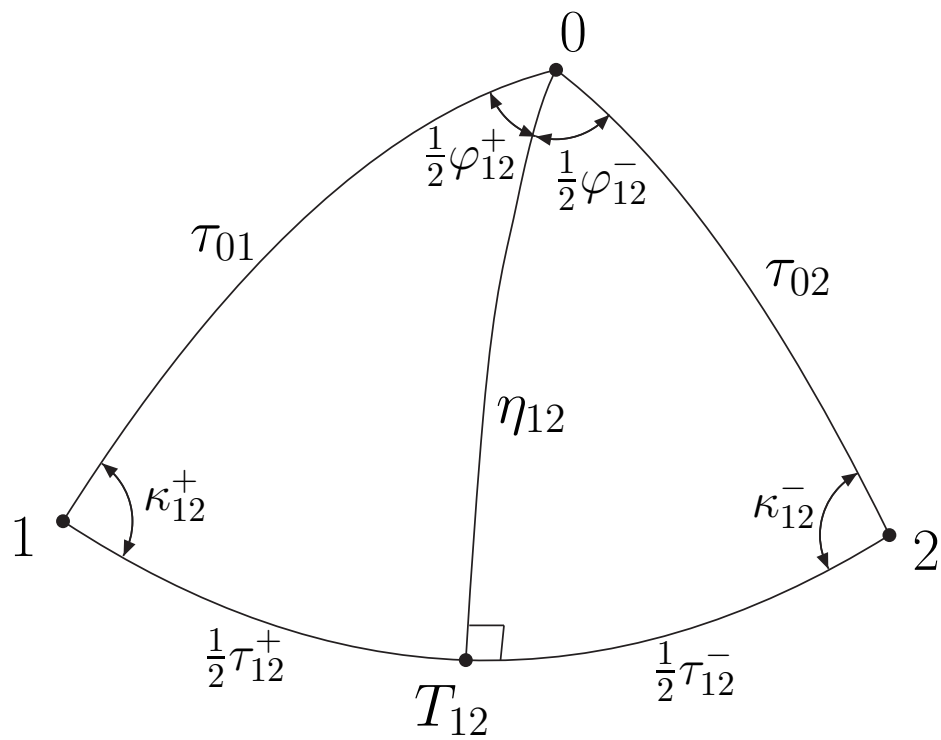

Fig. 9. An asymmetric spherical triangle 012 


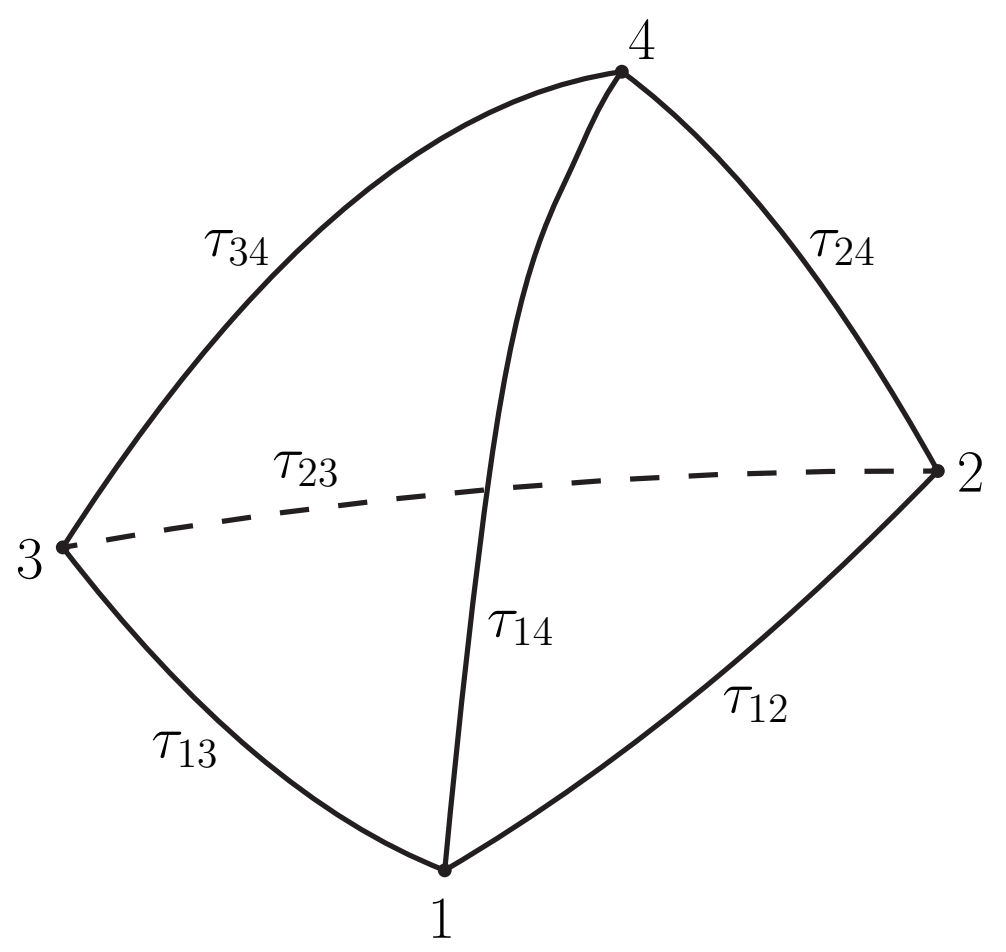

Fig. 10. The spherical tetrahedon 1234

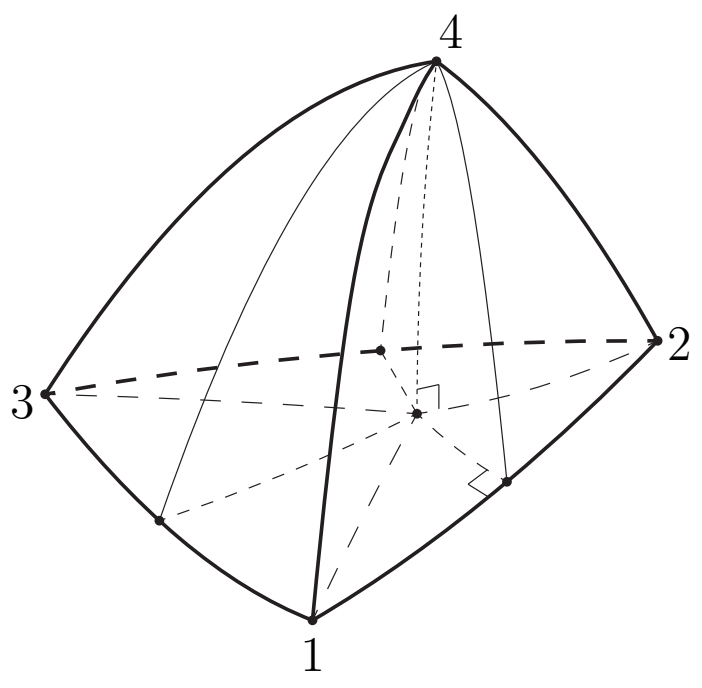

(a)

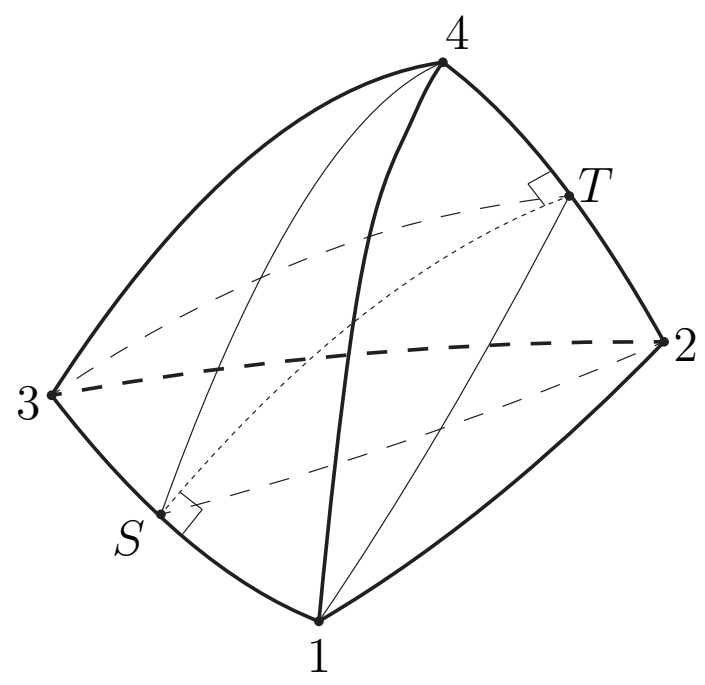

(b)

Fig. 11. Different ways of splitting the non-Euclidean tetrahedron 
UTAS-PHYS-97-12

hep-th/9709216

September 1997

\title{
A geometrical angle on Feynman integrals
}

\author{
A. I. Davydychev ${ }^{a, b, \text {, and R. Delbourgo }}{ }^{a, \text { }}$ \\ ${ }^{a}$ Physics Department, University of Tasmania, \\ GPO Box 252-21, Hobart, Tasmania, 7001 Australia \\ ${ }^{b}$ Institute for Nuclear Physics, Moscow State University, \\ 119899, Moscow, Russia
}

\begin{abstract}
A direct link between a one-loop $N$-point Feynman diagram and a geometrical representation based on the $N$-dimensional simplex is established by relating the Feynman parametric representations to the integrals over contents of $(N-1)$ dimensional simplices in non-Euclidean geometry of constant curvature. In particular, the four-point function in four dimensions is proportional to the volume of a three-dimensional spherical (or hyperbolic) tetrahedron which can be calculated by splitting into birectangular ones. It is also shown that the known formula of reduction of the $N$-point function in $(N-1)$ dimensions corresponds to splitting the related $N$-dimensional simplex into $N$ rectangular ones.
\end{abstract}

Published in J. Math. Phys. 39 (1998) 4299-4334

\footnotetext{
1'davyd@theory.npi.msu.su

2Bob.Delbourgo@utas.edu.au
} 


\section{Introduction}

The development of techniques for efficient calculation of one-loop $N$-point Feynman diagrams is very important for studying the leading and next-to-leading corrections to elementary particle processes within and beyond the Standard Model. Since any one-loop diagram can be reduced to a combination of scalar integrals [1], in what follows we shall mainly deal with scalar Feynman integrals.

It is well known (see e.g. in [2]) that results for the three- and four-point functions in four dimensions can be expressed in terms of dilogarithms (or related functions). The first explicit calculation of the general one-loop four-point function was given in [3] and later on, more compact results were presented in refs. [2, 4] (see also in [5, 6]). Moreover, it is known that (in four dimensions) the five-point function, the "pentagon", can be reduced to a linear combination of four-point functions [7, 8, 9] (see also in [10, 11, 12, 13]). Using linear dependence of the external momenta, a similar reduction procedure can be applied to the $N$-point functions with $N \geq 6$ (see in [14, 8]).

As a rule, explicit results for diagrams with several external legs possess a rather complicated analytical structure. For example, separate terms in a sum of dilogarithms may have singularities (cuts) which cancel in the whole sum. As the result, there are certain difficulties in describing analytic continuation to all regions (in external momenta and masses) of interest.

Another approach to the evaluation of one-loop integrals [15] makes it possible to represent them in terms of multiple hypergeometric functions. In ref. [16], such results for the integrals with an arbitrary number of external legs were presented. As a rule, the corresponding hypergeometric series have a rather restricted region of convergence. In general, the problem of analytic continuation of occurring functions to all other regions of interest is very complicated.

The analytical structure of the results can be better understood if one employs a geometrical interpretation of kinematic invariants and other quantities. For example, the singularities of the general three-point function can be described pictorially through a tetrahedron constructed out of the external and internal momenta. This method can be used to derive Landau equations defining the positions of possible singularities [17] (see also in [18, 19, 20]) and a similar approach can be applied to the four-point function [21, 3] too.

Another well-known example of using geometrical ideas is the massless three-point function with arbitrary (off-shell) external momenta. Let us denote them as $k_{12}, k_{23}$ and $k_{31}$, so that $k_{12}+k_{23}+k_{31}=0$, and assume that all these momenta are time-like $\left(k_{j l}^{2}>0\right)$. Using the standard notation for the "triangle" Källen function,

$$
\lambda(x, y, z)=x^{2}+y^{2}+z^{2}-2 x y-2 y z-2 z x,
$$

and assuming that we are in the region $\lambda\left(k_{12}^{2}, k_{23}^{2}, k_{31}^{2}\right) \leq 0$, the result for the corresponding one-loop scalar Feynman integral (in four dimensions) can be neatly expressed

\footnotetext{
${ }^{3}$ Here we use the same normalization as in (2.1). The results for the massless three-point functions can be found e.g. in [22, 2, 23]. They are closely connected with the results for two-loop massive vacuum diagrams [24, 25, 26].
} 
ast

$$
\frac{2 \mathrm{i} \pi^{2}}{\sqrt{-\lambda\left(k_{12}^{2}, k_{23}^{2}, k_{31}^{2}\right)}}\left\{\mathrm{Cl}_{2}\left(2 \theta_{12}\right)+\mathrm{Cl}_{2}\left(2 \theta_{23}\right)+\mathrm{Cl}_{2}\left(2 \theta_{31}\right)\right\}
$$

where the angles $\theta_{12}, \theta_{23}$ and $\theta_{31}$ are nothing but those of the triangle with sides $\sqrt{k_{12}^{2}}$, $\sqrt{k_{23}^{2}}$ and $\sqrt{k_{31}^{2}}$, as shown in Fig. 1. Moreover, the denominator $\sqrt{-\lambda}$ is nothing but four times the area of this triangle.

In this paper, we discuss the geometrical interpretation of the kinematic variables related to the one-loop $N$-point functions. The generalization of the tetrahedron representation (which was used in the three-point case) is an $N$-dimensional simplex. Furthermore, we show that there is a direct transition from the Feynman parametric representation to the geometrical description connected with an $N$-dimensional simplex. We thereby arrive at a "geometrical" way of evaluating Feynman integrals.

The paper is organized as follows. In Section 2 we describe the connection of the $N$ point variables and the corresponding $N$-dimensional simplex. In Section 3 we show how one can "geometrize" the ordinary Feynman parametric representation. In Sections 4,5,6 we consider application of these geometrical ideas to the two-, three- and four-point functions, respectively. In Section 7 we discuss how to use geometrical ideas for the reduction of $N$-point integrals in $(N-1)$ dimensions, and also for the calculation of the integrals with higher powers of denominators. Finally, in the last Section 8, we summarize the main ideas and results.

\section{Basic simplices in $N$ dimensions}

\section{1 $\quad N$-point function and the related simplex}

Consider a one-loop scalar $N$-point "sun-type" diagram presented in Fig. 2. The corresponding Feynman integral is

$$
J^{(N)}\left(n ; \nu_{1}, \ldots, \nu_{N}\right) \equiv \int \frac{\mathrm{d}^{n} q}{\prod_{i=1}^{N}\left[\left(p_{i}+q\right)^{2}-m_{i}^{2}\right]^{\nu_{i}}},
$$

where $n$ is the space-time dimension and $\nu_{i}$ are the powers of the propagators 9 . In general, it depends on $\frac{1}{2} N(N-1)$ momenta invariants $k_{j l}^{2}(j<l)$, where

$$
k_{j l} \equiv p_{j}-p_{l},
$$

and $N$ masses $m_{i}$ corresponding to the internal propagators. The momenta $p_{i}$ are auxiliary in the sense that they all can be shifted by a constant vector without changing the momenta $k_{j l}(2.2)$. We shall discuss this freedom below (see eqs. (2.49)-(2.53)).

\footnotetext{
${ }^{4}$ The functions related to the dilogarithm (including the Clausen function $\mathrm{Cl}_{2}(\theta)$ ) are defined in Appendix A (see also in 27]).

${ }^{5}$ Here and below, the usual causal prescription for the propagators is understood, i.e. $1 /\left[q^{2}-m^{2}\right]^{\nu} \leftrightarrow$ $1 /\left[q^{2}-m^{2}+\mathrm{i} 0\right]^{\nu}$.
} 
It is well known that, using the condition $\sum \alpha_{i}=1$, the standard quadratic form occurring in the denominator of the integrand of the Feynman parametric representation for these integrals (cf. eqs. (3.1)-(3.2) below) can be rewritten in a homogeneous form,

$$
\left[\sum_{j<l} \alpha_{j} \alpha_{l} k_{j l}^{2}-\sum \alpha_{i} m_{i}^{2}\right] \Rightarrow-\left[\sum \alpha_{i}^{2} m_{i}^{2}+2 \sum_{j<l} \sum_{j} \alpha_{l} m_{j} m_{l} c_{j l}\right],
$$

where $0^{\circ}$

$$
c_{j l} \equiv \frac{m_{j}^{2}+m_{l}^{2}-k_{j l}^{2}}{2 m_{j} m_{l}} .
$$

In the region between the corresponding two-particle pseudo-threshold, $k_{j l}^{2}=\left(m_{j}-m_{l}\right)^{2}$, and the threshold, $k_{j l}^{2}=\left(m_{j}+m_{l}\right)^{2}$, we have $\left|c_{j l}\right|<1$, and therefore in this region they can be understood as cosines of some angles $\tau_{j l}$,

$$
c_{j l}=\cos \tau_{j l}=\left\{\begin{array}{rl}
1, & k_{j l}^{2}=\left(m_{j}-m_{l}\right)^{2} \\
-1, & k_{j l}^{2}=\left(m_{j}+m_{l}\right)^{2}
\end{array} .\right.
$$

The corresponding angles $\tau_{j l}$ are

$$
\tau_{j l}=\arccos \left(c_{j l}\right)=\arccos \left(\frac{m_{j}^{2}+m_{l}^{2}-k_{j l}^{2}}{2 m_{j} m_{l}}\right)=\left\{\begin{array}{ll}
0, & k_{j l}^{2}=\left(m_{j}-m_{l}\right)^{2} \\
\pi, & k_{j l}^{2}=\left(m_{j}+m_{l}\right)^{2}
\end{array} .\right.
$$

The expressions in other regions should be understood in the sense of analytic continuation, using (when necessary) the causal prescription for the propagators. For example, when $k_{j l}^{2}<\left(m_{j}-m_{l}\right)^{2}\left(c_{j l}>1\right)$, i.e. below the pseudothreshold, we should interpret

$$
\tau_{j l}=-\mathrm{i} \operatorname{Arch}\left(c_{j l}\right)=-\frac{\mathrm{i}}{2} \ln \left(\frac{m_{j}^{2}+m_{l}^{2}-k_{j l}^{2}+\sqrt{\lambda\left(m_{j}^{2}, m_{l}^{2}, k_{j l}^{2}\right)}}{m_{j}^{2}+m_{l}^{2}-k_{j l}^{2}-\sqrt{\lambda\left(m_{j}^{2}, m_{l}^{2}, k_{j l}^{2}\right)}}\right),
$$

where $\lambda(x, y, z)$ is the Källen function defined by eq. (1.1). When $k_{j l}^{2}>\left(m_{j}+m_{l}\right)^{2}$ $\left(c_{j l}<-1\right)$, i.e. above the threshold, we get

$$
\tau_{j l}=\pi+\mathrm{i} \operatorname{Arch}\left(-c_{j l}\right)=\pi+\frac{\mathrm{i}}{2} \ln \left(\frac{k_{j l}^{2}-m_{j}^{2}-m_{l}^{2}+\sqrt{\lambda\left(m_{j}^{2}, m_{l}^{2}, k_{j l}^{2}\right)}}{k_{j l}^{2}-m_{j}^{2}-m_{l}^{2}-\sqrt{\lambda\left(m_{j}^{2}, m_{l}^{2}, k_{j l}^{2}\right)}}\right) .
$$

Note that $\lambda\left(m_{j}^{2}, m_{l}^{2}, k_{j l}^{2}\right)$ is positive when $k_{j l}^{2}<\left(m_{j}-m_{l}\right)^{2}$ or $k_{j l}^{2}>\left(m_{j}+m_{l}\right)^{2}$.

When the angles $\tau_{j l}$ (defined by eq. (2.6)) are real, the quadratic form on the r.h.s. in eq. (2.3) has a rather simple geometrical interpretation. Let us consider a set of Euclidean "mass" vectors whose lengths are $m_{i}$. Let them be directed so that the angle between the $j$-th and the $l$-th vectors is $\tau_{j l}$. If we denote the corresponding unit vectors as $a_{i}$ (so that the "mass" vectors are $\left.m_{i} a_{i}\right)$, we get

$$
\left(a_{j} \cdot a_{l}\right)=\cos \tau_{j l}=c_{j l} .
$$

\footnotetext{
${ }^{6}$ In some papers, the notation $y_{j l}$ is used for $\pm c_{j l}$.
} 
This is also valid for the case $j=l$, since $\tau_{j j}=0$ and $c_{j j}=1$, cf. eqs. (2.5)-(2.6). Now, we can represent eq. (2.3) as

$$
\sum \alpha_{i}^{2} m_{i}^{2}+2 \sum_{j<l} \alpha_{j} \alpha_{l} m_{j} m_{l} c_{j l}=\left(\sum \alpha_{i} m_{i} a_{i}\right)^{2}
$$

In $N$ dimensions, if we put all "mass" vectors together as emanating from a common origin, they, together with the sides connecting their ends, will define a simplex (or hypertetrahedron) which is the basic one for a given Feynman diagram. An example of such a simplex for $N=4$ is illustrated by Fig. 3.7 It is easy to see that the length of the side connecting the ends of the $j$-th and the $l$-th mass vectors is $\left(m_{j}^{2}+m_{l}^{2}-2 m_{j} m_{l} c_{j l}\right)^{1 / 2}=$ $\left(k_{j l}^{2}\right)^{1 / 2}$, so we shall call it a "momentum" side. In total, the basic $N$-dimensional simplex has $\frac{1}{2} N(N+1)$ sides, among them $N$ mass sides (corresponding to the masses $m_{1}, \ldots, m_{N}$ ) and $\frac{1}{2} N(N-1)$ momentum sides (corresponding to the momenta $k_{j l}, j<l$ ), which meet at $(N+1)$ vertices. Each vertex is a "meeting point" for $N$ sides. There is one vertex where all mass sides meet, which we shall call the mass meeting point, $M$. All other vertices are meeting points for $(N-1)$ momentum sides and one mass side. Furthermore, the number of $(N-1)$-dimensional hyperfaces is $(N+1)$. $N$ of them (the reduced hyperfaces) can be obtained by excluding one mass side in turn, together with the corresponding vertex and the momentum sides meeting at that vertex. The reduced hyperface without the $j$-th mass side is nothing but the $(N-1)$-dimensional simplex corresponding to an $(N-1)$ point function obtained from the $N$-point function by shrinking the $j$-th propagator; this is equivalent to the power of propagator vanishing, $\nu_{j}=0$. The last hyperface, namely the momentum hyperface involves all momentum sides but does not include any mass sides. It is associated with the massless $N$-point function possessing the same external momenta as the basic one.

In general, in the $N$-point function only $(N-1)$ external momenta are independent, because they are related via the conservation law. We can choose, for example, the vectors $k_{1 N}, k_{2 N}, \ldots, k_{N-1, N}$. Considering all scalar products of these momenta, including their squares, we get $\frac{1}{2} N(N-1)$ invariants, as stated above. However, when $n$ is integer and less than $(N-1)$, only $n$ of the $(N-1)$ momenta are independent, e.g. $k_{1 N}, k_{2 N}, \ldots, k_{n N}$. Each of the remaining $(N-n-1)$ vectors $k_{j N}(j=n+1, \ldots, N-1)$ can be fixed by $n$ scalar products $\left(k_{j N} \cdot k_{l N}\right)(l=1, \ldots, N-1)$. Hence the total number of independent momentum invariants for integer $n \leq N-1$ is

$$
\frac{1}{2} n(n+1)+n(N-n-1)=\frac{1}{2} n(2 N-n-1),
$$

while for $n \geq N-1$ it remains equal to $\frac{1}{2} N(N-1)$; both expressions coincide for $n=N-1$. The linear dependence between the external momenta reveals itself in the degeneracy of the results associated with the vanishing of certain hypervolumes (see below).

We note that there is a difference between the "real" momenta of the $N$-point diagram, $k_{j l}$, and the vectors

$$
K_{j l} \equiv m_{j} a_{j}-m_{l} a_{l},
$$

\footnotetext{
${ }^{7}$ Since the four-dimensional Euclidean space is understood, one should consider Fig. 3 just as an illustration (rather than precise picture).
} 
corresponding to the momentum sides of the simplex (the same applies to the momenta $p_{i}$ and $P_{i}$ discussed in subsection 2.4). The momenta $k_{j l}$ are defined in pseudo-Euclidean (Minkowski) space, and they are time-like $\left(k_{j l}^{2}>0\right)$ in the region between the threshold and pseudo-threshold. The momentum sides of the simplex, $K_{j l}$, are vectors in Euclidean space, the (Euclidean) squares of their lengths $\left(K_{j l}^{2}\right)$ being equal to $k_{j l}^{2}$. In what follows, we shall consider the $K_{j l}$ as Euclidean analogues of the $k_{j l}$ and, vice versa, the $k_{j l}$ are pseudo-Euclidean analogues of the $K_{j l}$. Since the $k_{j l}^{2}(j<l)$ form the complete basis of external invariants, all invariant scalars in Minkowski and Euclidean spaces are the same.

The matrix with the components (2.4),

$$
\|c\| \equiv\left\|c_{j l}\right\| \equiv\left(\begin{array}{ccccc}
1 & c_{12} & c_{13} & \ldots & c_{1 N} \\
c_{12} & 1 & c_{23} & \ldots & c_{2 N} \\
c_{13} & c_{23} & 1 & \ldots & c_{3 N} \\
\ldots \ldots & \ldots & \ldots & \ldots & \ldots \\
c_{1 N} & c_{2 N} & c_{3 N} & \ldots & 1
\end{array}\right),
$$

is associated with many geometrical properties of the basic simplexf. In particular, we shall need its determinant,

$$
D^{(N)} \equiv \operatorname{det}\left\|c_{j l}\right\|
$$

and the minors

$D_{j l}^{(N-1)} \equiv\{$ minor of $(2.13)$ obtained by eliminating the $j$-th row and the $l$-th column $\}$.

In particular, when $j=l$ the minor $D_{j j}^{(N-1)}$ is a principal one for the matrix (2.13).

The formulae for the content or hypervolume of the basic $N$-dimensional simplex and its hyperfaces, in terms of the determinant (2.14) and the minors (2.15), are well known in the $N$-dimensional Euclidean geometry (see for instance [28, 29]). Thus the content of the $N$-dimensional simplex is given by

$$
V^{(N)}=\frac{1}{N !}\left(\prod_{i=1}^{N} m_{i}\right) \sqrt{D^{(N)}} .
$$

The content of the $j$-th $(N-1)$-dimensional reduced hyperface (which does not contain the $j$-th mass side) is

$$
\bar{V}_{j}^{(N-1)}=\frac{1}{(N-1) !}\left(\prod_{i \neq j} m_{i}\right) \sqrt{D_{j j}^{(N-1)}}
$$

whereas the content of the $(N-1)$-dimensional momentum hyperface is

$$
\bar{V}_{0}^{(N-1)}=\frac{1}{(N-1) !} \sqrt{\Lambda^{(N)}}
$$

where $\Lambda^{(N)}$ is symmetric combination of the momenta defined in the same way as in [1],

$$
\Lambda^{(N)} \equiv \operatorname{det}\left\|\left(k_{j N} \cdot k_{l N}\right)\right\|
$$

\footnotetext{
${ }^{8}$ The matrix $(2.13)$ is nothing but the Gram matrix of the vectors $a_{1}, \ldots, a_{N}$.
} 
Note that the content of the basic simplex can also be presented as

$$
V^{(N)}=\frac{1}{N} \bar{V}_{0}^{(N-1)} m_{0},
$$

where $m_{0}$ is the distance between the mass meeting point and the momentum hyperface, i.e. the length of the vector of the height of the simplex, $H_{0}$. Therefore,

$$
m_{0} \equiv\left|H_{0}\right|=N \frac{V^{(N)}}{\bar{V}_{0}^{(N-1)}}=\left(\prod_{i=1}^{N} m_{i}\right) \sqrt{\frac{D^{(N)}}{\Lambda^{(N)}}} .
$$

\subsection{Normals, dihedral angles and the dual matrix}

The dihedral angles between the hyperfaces can be defined via the angles between their normals. Thus, we get $\frac{1}{2} N(N-1)$ dihedral angles between pairs of the reduced hyperfaces and $N$ angles between the momentum hyperface and the reduced ones.

Again, let $a_{i}$ be unit vectors directed along the mass sides of the basic $N$-dimensional simplex. The normals to the $(N-1)$-dimensional reduced hyperfaces can be defined as

$$
n_{j \lambda}=\frac{\partial}{\partial a_{j}^{\lambda}}\left(\epsilon_{\nu_{1} \ldots \nu_{N}} a_{1}^{\nu_{1}} \ldots a_{N}^{\nu_{N}}\right) .
$$

where $\epsilon_{\nu_{1} \ldots \nu_{N}}$ is the completely antisymmetric tensor in $N$ dimensions.

Using the well-known expression for the contraction of the product of two $\epsilon$ tensors in terms of the determinant with Kronecker $\delta$ symbols, it is easy to show that

$$
\left(n_{j} \cdot n_{l}\right)=(-1)^{j+l} D_{j l}^{(N-1)},
$$

where $D_{j l}^{(N-1)}$ is defined in (2.15). In particular, $n_{j}^{2}$ is nothing but the principal minor $D_{j j}^{(N-1)}$ related to the $(N-1)$-point function associated with the corresponding reduced hyperface. The cosine of the angle $\widetilde{\tau}_{j l}$ between the normals to the $j$-th and the $l$-th reduced hyperfaces is

$$
\widetilde{c}_{j l} \equiv \cos \widetilde{\tau}_{j l}=\frac{\left(n_{j} \cdot n_{l}\right)}{\sqrt{n_{j}^{2} n_{l}^{2}}}=\frac{(-1)^{j+l} D_{j l}^{(N-1)}}{\sqrt{D_{j j}^{(N-1)} D_{l l}^{(N-1)}}} .
$$

In particular, this means that the matrix with the elements $\sqrt{D_{j j}^{(N-1)} D_{l l}^{(N-1)}} \widetilde{c}_{j l} / D^{(N)}$ is the inverse of the matrix (2.13). Namely,

$$
\left\|c_{j l}\right\|^{-1}=\frac{1}{D^{(N)}} \operatorname{diag}\left(\sqrt{D_{11}^{(N-1)}}, \ldots, \sqrt{D_{N N}^{(N-1)}}\right)\left\|\widetilde{c}_{j l}\right\| \operatorname{diag}\left(\sqrt{D_{11}^{(N-1)}}, \ldots, \sqrt{D_{N N}^{(N-1)}}\right)
$$

where $D^{(N)}$ is defined in (2.14). We shall call the matrix $\left\|\widetilde{c}_{j l}\right\|$ the dual matrix (with respect to $\left.\left\|c_{j l}\right\|\right)$. A trivial corollary of $(2.25)$ is that

$$
\widetilde{D}^{(N)} \equiv \operatorname{det}\left\|\widetilde{c}_{j l}\right\|=\frac{\left(D^{(N)}\right)^{N-1}}{\prod_{i=1}^{N} D_{i i}^{(N-1)}} .
$$

\footnotetext{
${ }^{9}$ The matrix $\left\|\widetilde{c}_{j l}\right\|$ is nothing but the Gram matrix of unit vectors directed along the normals $n_{i}$ $(i=1, \ldots, N)$.
} 
Note that the dihedral angles $\psi_{j l}$ are related to the angles $\widetilde{\tau}_{j l}$ as

$$
\psi_{j l}=\pi-\widetilde{\tau}_{j l}, \quad \cos \psi_{j l}=-\cos \widetilde{\tau}_{j l} .
$$

The normal to the momentum hyperface can be defined as

$$
n_{0 \lambda}=-\epsilon_{\mu_{1} \ldots \mu_{N-1} \lambda}\left(K_{1 N}\right)^{\mu_{1}} \ldots\left(K_{N-1, N}\right)^{\mu_{N-1}} .
$$

If we represent $K_{j N}=\left(m_{j} a_{j}-m_{N} a_{N}\right)$ and use the fact that all terms involving two or more $m_{N} a_{N}$ disappear in (2.28) (due to the antisymmetry of the $\epsilon$ tensor), we get the following representation:

$$
n_{0 \lambda}=-\left(\prod_{i=1}^{N} m_{i}\right) \sum_{j=1}^{N} \frac{n_{j \lambda}}{m_{j}} .
$$

On one hand, the original definition $(2.28)$ leads to

$$
n_{0}^{2}=\operatorname{det}\left\|\left(k_{j N} \cdot k_{l N}\right)\right\| \equiv \Lambda^{(N)}
$$

where $\Lambda^{(N)}$ is defined in (2.19). On the other hand, using (2.29) we obtain

$$
n_{0}^{2}=\left(\prod_{i=1}^{N} m_{i}^{2}\right) \sum_{l=1}^{N} \frac{1}{m_{l}^{2}} F_{l}^{(N)}
$$

where (cf. eq. (4) of [11])

$$
F_{l}^{(N)}=\sum_{j=0}^{N}(-1)^{j+l} D_{j l}^{(N-1)} \frac{m_{l}}{m_{j}}=\frac{\partial}{\partial m_{l}^{2}}\left(m_{l}^{2} D^{(N)}\right),
$$

i.e. $F_{l}^{(N)}$ is the determinant obtained from det $\left\|c_{j l}\right\|$ by substituting, instead of the $l$-th column, the mass ratios $m_{l} / m_{j}$ (where $j$ is the line number). When taking the partial derivative in (2.32), it is implied that the set of independent variables involves $m_{i}^{2}$ and $k_{j l}^{2}$, rather than $c_{j l}$. In particular, the representation of $F_{l}^{(N)}$ in terms of determinants (2.32) (see also in [11]) shows that they obey the following set of linear equations:

$$
\sum_{l=1}^{N} c_{j l} F_{l}^{(N)} \frac{1}{m_{l}}=D^{(N)} \frac{1}{m_{j}} .
$$

Another useful representation of $n_{0}$ is

$$
n_{0}=-\frac{\prod m_{l}}{\sqrt{D^{(N)}}} \sum_{i=1}^{N} \frac{1}{m_{i}} F_{i}^{(N)} a_{i},
$$

where, as usually, $a_{i}$ are the unit vectors directed along the mass sides of the basic simplex. Using eq. (2.33) (and remembering that $\left(a_{j} \cdot a_{l}\right)=c_{j l}$ ), one can easily see that the representation (2.34) is equivalent to eqs. (2.28) and (2.29). Namely, $n_{0}$ (defined by (2.34)) is orthogonal to all $K_{j l}=m_{j} a_{j}-m_{l} a_{l}$ and $n_{0}^{2}$ is the same as in eq. (2.31). 


\subsection{Splitting the basic simplex}

The geometrical meaning of $F_{l}^{(N)}$ can be understood as follows. Using eqs. (2.29) and (2.23), we get

$$
\left(n_{0} \cdot n_{l}\right)=-\left(\prod_{i \neq l} m_{i}\right) F_{l}^{(N)}
$$

Therefore, the cosine of the angle $\widetilde{\tau}_{0 l}$ between the normals to the momentum hyperface and the $l$-th reduced hyperface is

$$
\widetilde{c}_{0 l} \equiv \cos \widetilde{\tau}_{0 l}=\frac{\left(n_{0} \cdot n_{l}\right)}{\sqrt{n_{0}^{2} n_{l}^{2}}}=-\frac{\left(\prod_{i \neq l} m_{i}\right) F_{l}^{(N)}}{\sqrt{\Lambda^{(N)} D_{l l}^{(N-1)}}},
$$

whereas the dihedral angle between these hyperfaces is given by

$$
\psi_{0 l}=\pi-\widetilde{\tau}_{0 l}, \quad \cos \psi_{0 l}=-\cos \widetilde{\tau}_{0 l} .
$$

The orthogonal projection of the $i$-th reduced hyperface onto the momentum hyperface is

$$
\bar{V}_{i}^{(N-1)} \cos \psi_{0 i} \text {. }
$$

The sum of all such projections should cover the whole momentum hyperface,

$$
\sum_{i=1}^{N} \bar{V}_{i}^{(N-1)} \cos \psi_{0 i}=\bar{V}_{0}^{(N-1)}
$$

Using eqs. (2.36), (2.17) and (2.18), we see that the condition (2.39) is equivalent to

$$
\left(\prod_{j=1}^{N} m_{j}^{2}\right) \sum_{l=1}^{N} \frac{F_{l}^{(N)}}{m_{l}^{2}}=\Lambda^{(N)}
$$

which is the case, cf. eqs. (2.30), (2.31). The condition (2.40) is equivalent to eq. (38) of [11] (see also in [3]). Eq. (2.39) illustrates the geometrical meaning of (2.40). Moreover, we can see that, if we use the "height" $H_{0}$ for splitting the basic $N$-dimensional simplex into $N$ rectangular ones whose "bases" correspond to the projections (2.38), the content of the $i$-th rectangular simplex $V_{i}^{(N)}$ is proportional to $F_{i}^{(N)}$, namely

$$
V_{i}^{(N)}=\frac{1}{N} \bar{V}_{i}^{(N-1)} m_{0} \cos \psi_{0 i}=\frac{V^{(N)}}{\Lambda^{(N)}}\left(\prod_{l \neq i} m_{l}^{2}\right) F_{i}^{(N)} .
$$

In the same manner we can consider a projection onto one of the reduced hyperfaces. This gives

$$
\sum_{l \neq i} \bar{V}_{l}^{(N-1)} \cos \psi_{i l}+\bar{V}_{0}^{(N-1)} \cos \psi_{0 i}=\bar{V}_{i}^{(N-1)}
$$

or

$$
\sum_{l=1}^{N} \bar{V}_{l}^{(N-1)} \widetilde{c}_{i l}+\bar{V}_{0}^{(N-1)} \widetilde{c}_{0 i}=0
$$


In terms of $F_{l}^{(N)}$, eq. (2.43) corresponds to the definition (2.32).

The cosine of the angle $\tau_{0 j}$ between the $j$-th mass side and the height $H_{0}$ is

$$
c_{0 j} \equiv \cos \tau_{0 j}=\frac{m_{0}}{m_{j}} .
$$

Consider the matrix of cosines (similar to (2.13)) associated with the $i$-th rectangular simplex. Its elements are

$$
\left\{\begin{array}{cc}
c_{j l}, & \text { if } j \neq i \text { and } l \neq i \\
c_{0 l}, & \text { if } j=i \text { and } l \neq i \\
c_{0 j}, & \text { if } l=i \text { and } j \neq i \\
1, & \text { if } j=l=i
\end{array}\right.
$$

Denoting

$$
D_{i}^{(N)} \equiv\{\text { determinant of the matrix }(2.45)\},
$$

and using eqs. (2.16) and (2.21), we get

$$
V_{i}^{(N)}=\left(\prod_{l \neq i} m_{l}\right) \sqrt{\frac{D_{i}^{(N)}}{\Lambda^{(N)}}} .
$$

Comparing eqs. (2.41) and (2.47), we obtain the following result for the determinant (2.46):

$$
D_{i}^{(N)}=\left(\prod_{l \neq i} m_{l}\right) \frac{\left(F_{i}^{(N)}\right)^{2}}{\Lambda^{(N)}} .
$$

\subsection{Choice of the momenta $p_{i}$}

Let us discuss possibilities for a choice of the momenta $p_{i}$ in eq. (2.1) and their Euclidean analogues $P_{i}$, such that $K_{j l}=P_{j}-P_{l}$. Due to eq. (2.2), the $p_{i}$ vectors emanating from an origin, together with the vectors $k_{j l}$, form an $N$-dimensional simplex in pseudo-Euclidean space 1 . Analogously, in Euclidean space the vectors $P_{i}$ together with the $K_{j l}$ momentum hyperface (taken as a base), form an $N$-dimensional Euclidean simplex similar to the basic one. Using translational invariance of $p_{i}$, we can shift their origin as we like. In particular, we can make one of the $p_{i}$ vectors zero, whereupon the origin of $P_{i}$ coincides with one of the vertices belonging to the momentum hyperface. However, this way would break the symmetry of the problem. There are at least two reasonable, symmetric ways to fix the origin of $p_{i}\left(\right.$ and $\left.P_{i}\right)$ :

1. Choose the mass meeting point as the origin of $P_{i}$ vectors, so that $P_{i}^{2}=p_{i}^{2}=m_{i}^{2}$ and the $P_{i}$ vectors coincide with the mass sides, $P_{i}=m_{i} a_{i}$. In this case, the basic simplex becomes even "more basic", and the integral (2.1) can be expressed as

$$
J^{(N)}\left(n ; \nu_{1}, \ldots, \nu_{N}\right)=\left.\int \frac{\mathrm{d}^{n} q}{\prod_{i=1}^{N}\left[q^{2}+2\left(p_{i} \cdot q\right)\right]^{\nu_{i}}}\right|_{\left(p_{j} \cdot p_{l}\right)=m_{j} m_{l} c_{j l}} .
$$

\footnotetext{
${ }^{10}$ For integer $n<N$, this simplex is degenerate, since one cannot use more than $n$ dimensions for external vectors.
} 
Such a choice of $p_{i}$ simplifies study of singularities $\square$ via Landau equations [17, 20]. Note that, according to eq. (2.34), in this case the vector of the height $H_{0}$ can be represented as

$$
H_{0}=\frac{\prod m_{l}^{2}}{\Lambda^{(N)}} \sum_{i=1}^{N} \frac{1}{m_{i}^{2}} F_{i}^{(N)} P_{i} .
$$

However, this choice of $p_{i}$ cannot be used for integer $n<N$.

2. Pick, as the origin of $P_{i}$ vectors, the point of intercept of the height $H_{0}$ and the momentum hyperface. This is nothing but a projection of the previous option onto the momentum hyperface. In this case (cf. eq. (2.44)),

$$
\begin{aligned}
p_{i}^{2} & =P_{i}^{2}=m_{i}^{2}-m_{0}^{2}=m_{i}^{2}\left(1-c_{0 i}^{2}\right), \\
\left(p_{j} \cdot p_{l}\right) & =\left(P_{j} \cdot P_{l}\right)=m_{j} m_{l} c_{j l}-m_{0}^{2}=m_{j} m_{l}\left(c_{j l}-c_{0 j} c_{0 l}\right),
\end{aligned}
$$

and the denominators in the integral (2.1) become

$$
\left[\left(p_{i}+q\right)^{2}-m_{i}^{2}\right] \Rightarrow\left[q^{2}+2\left(p_{i} \cdot q\right)-m_{0}^{2}\right] \text {. }
$$

The projection of eq. 2.34) onto the momentum hyperface shows that there exists the following relation which is valid for this choice of $P_{i}$ :

$$
\sum_{i=1}^{N} \frac{1}{m_{i}^{2}} F_{i}^{(N)} P_{i}=0 .
$$

\section{From Feynman parameters to non-Euclidean ge- ometry}

\subsection{Moving from linear to quadratic hypersurface}

The standard Feynman parametric representation of a one-loop $N$-point integral in $n$ dimensions (2.1), corresponding to the diagram presented in Fig. 2, reads

$$
J^{(N)}\left(n ; \nu_{1}, \ldots, \nu_{N}\right)=\mathrm{i}^{1-n} \pi^{n / 2} \frac{\Gamma\left(\sum \nu_{i}-\frac{n}{2}\right)}{\prod \Gamma\left(\nu_{i}\right)} \int_{0}^{1} \ldots \int_{0}^{1} \frac{\prod \alpha_{i}^{\nu_{i}-1} \mathrm{~d} \alpha_{i} \delta\left(\sum \alpha_{i}-1\right)}{\left[\sum_{j<l} \sum_{j} \alpha_{l} k_{j l}^{2}-\sum \alpha_{i} m_{i}^{2}\right]^{\Sigma \nu_{i}-n / 2}} .
$$

As we have already mentioned (2.3), the integrand can be written in a homogeneous form:

$$
J^{(N)}\left(n ; \nu_{1}, \ldots, \nu_{N}\right)=\mathrm{i}^{1-2 \Sigma \nu_{i}} \pi^{n / 2} \frac{\Gamma\left(\sum \nu_{i}-\frac{n}{2}\right)}{\prod \Gamma\left(\nu_{i}\right)} \int_{0}^{1} \ldots \int_{0}^{1} \frac{\prod \alpha_{i}^{\nu_{i}-1} \mathrm{~d} \alpha_{i} \delta\left(\sum \alpha_{i}-1\right)}{\left[\sum \alpha_{i}^{2} m_{i}^{2}+2 \sum_{j<l} \sum_{j} \alpha_{j} \alpha_{l} m_{j} m_{l} c_{j l}\right]^{\Sigma \nu_{i}-n / 2}}
$$

where the "cosines" $c_{j l}$ are defined in (2.4). Note that the limits of integration in eqs. (3.1)(3.2) can be extended from $(0,1)$ to $(0, \infty)$, since the actual region of integration is defined

\footnotetext{
${ }^{11}$ In this case, the main Landau singularity is associated with $q \rightarrow 0$.
} 
by the $\delta$ function. It corresponds to a part of an $(N-1)$-dimensional hyperplane $\sum \alpha_{i}=1$ which is cut out by the conditions $\alpha_{i} \geq 0(i=1, \ldots, N)$.

Now, let us consider the integral (3.2), and let us use a rescaling which is similar to one used in 21, 2, 4] (see also in [25 where the transformations of a general form have been discussed). Let us rescale $\alpha_{i}=m_{i}^{-1} \alpha_{i}^{\prime}$, so that the $\delta$ function becomes

$$
\delta\left(\sum \frac{\alpha_{i}^{\prime}}{m_{i}}-1\right)
$$

To restore the argument of the $\delta$ function in its original form, let us substitute

$$
\alpha_{i}^{\prime}=\mathcal{F}\left(\alpha_{1}^{\prime \prime}, \ldots, \alpha_{N}^{\prime \prime}\right) \alpha_{i}^{\prime \prime}, \quad \text { with } \quad \mathcal{F}\left(\alpha_{1}, \ldots, \alpha_{N}\right)=\frac{\sum \alpha_{i}}{\sum \frac{\alpha_{i}}{m_{i}}} .
$$

Note that the Jacobian of this substitution is $\mathcal{F}^{N}$ (cf. Appendix B).

Suppressing the primes, we arrive at the following representation:

$$
J^{(N)}\left(n ; \nu_{1}, \ldots, \nu_{N}\right)=\mathrm{i}^{1-2 \Sigma \nu_{i}} \pi^{n / 2} \frac{\Gamma\left(\sum \nu_{i}-\frac{n}{2}\right)}{\prod \Gamma\left(\nu_{i}\right)} \frac{1}{\prod m_{i}^{\nu_{i}}} \int_{0}^{1} \ldots \int_{0}^{1} \frac{\prod \alpha_{i}^{\nu_{i}-1} \mathrm{~d} \alpha_{i} \delta\left(\sum \alpha_{i}-1\right)}{\left(\sum \frac{\alpha_{i}}{m_{i}}\right)^{n-\Sigma \nu_{i}}\left(\alpha^{T}\|c\| \alpha\right)^{\Sigma \nu_{i}-n / 2}},
$$

where we use matrix notation (2.13),

$$
\alpha^{T}\|c\| \alpha \equiv \sum_{j=1}^{N} \sum_{l=1}^{N} c_{j l} \alpha_{j} \alpha_{l}=\sum \alpha_{i}^{2}+2 \sum_{j<l} \alpha_{j} \alpha_{l} c_{j l}
$$

Next, let us change the variables from $\alpha$ to $\alpha^{\prime}$ via

$$
\alpha_{i}=\mathcal{G}\left(\alpha_{1}^{\prime}, \ldots, \alpha_{N}^{\prime}\right) \alpha_{i}^{\prime}, \quad \text { with } \quad \mathcal{G}\left(\alpha_{1}^{\prime}, \ldots, \alpha_{N}^{\prime}\right)=\frac{\sum\left(\alpha_{i}^{\prime}\right)^{2}+2 \sum_{j<l} \sum_{j} \alpha_{j}^{\prime} \alpha_{l}^{\prime} c_{j l}}{\sum \alpha_{i}^{\prime}} .
$$

Here, the Jacobian of the substitution is $2 \mathcal{G}^{N}$ (see Appendix B). Effectively, we put the quadratic form into the argument of the $\delta$ function. However, the linear denominator (coming from the $\mathcal{F}$ function) survives and we arrive at

$J^{(N)}\left(n ; \nu_{1}, \ldots, \nu_{N}\right)=2 \mathrm{i}^{1-2 \Sigma \nu_{i}} \pi^{n / 2} \frac{\Gamma\left(\sum \nu_{i}-\frac{n}{2}\right)}{\prod \Gamma\left(\nu_{i}\right)} \frac{1}{\prod m_{i}^{\nu_{i}}} \int_{0}^{\infty} \ldots \int_{0}^{\infty} \frac{\prod \alpha_{i}^{\nu_{i}-1} \mathrm{~d} \alpha_{i}}{\left(\sum \frac{\alpha_{i}}{m_{i}}\right)^{n-\Sigma \nu_{i}}} \delta\left(\alpha^{T}\|c\| \alpha-1\right)$,

where we continue to use the matrix notation (3.6).

\subsection{The case $\sum \nu_{i}=n$}

In the particular case $\sum \nu_{i}=n$ the linear denominator disappears and eq. (3.5) gives

$$
\left.J^{(N)}\left(n ; \nu_{1}, \ldots, \nu_{N}\right)\right|_{\Sigma \nu_{i}=n}=\mathrm{i}^{1-2 \Sigma \nu_{i}} \pi^{n / 2} \frac{\Gamma\left(\frac{n}{2}\right)}{\prod \Gamma\left(\nu_{i}\right)} \frac{1}{\prod m_{i}^{\nu_{i}}} \int_{0}^{1} \ldots \int_{0}^{1} \frac{\prod \alpha_{i}^{\nu_{i}-1} \mathrm{~d} \alpha_{i} \delta\left(\sum \alpha_{i}-1\right)}{\left(\alpha^{T}\|c\| \alpha\right)^{\Sigma \nu_{i}-n / 2}} .
$$


Here all dependence on the external momenta and masses in the integral is through $c_{j l}$, eq. (2.4).

Since the final integral (3.9) is the same as in the equal-mass case, we can formulate the following statement: Let $\sum \nu_{i}=n$, and let the result for the Feynman integral (3.1) with equal masses $\left(m_{i}=m, i=1, \ldots, N\right)$ be represented by a dimensionless function $\Psi$ depending on the quantities $\overline{\bar{c}}_{j l} \equiv 1-k_{j l}^{2} /\left(2 m^{2}\right)$ (corresponding to eq. (2.4) in the equal-mass case) as

$$
\left.J^{(N)}\left(n ; \nu_{1}, \ldots, \nu_{N}\right)\right|_{\Sigma \nu_{i}=n, m_{i}=m}=\frac{1}{m^{\Sigma \nu_{i}}} \Psi\left(\left\{\overline{\bar{c}}_{j l}\right\}\right)
$$

Then the result for the corresponding integral with different masses $m_{i}$ can be expressed in terms of the same function $\Psi$ as

$$
\left.J^{(N)}\left(n ; \nu_{1}, \ldots, \nu_{N}\right)\right|_{\Sigma \nu_{i}=n}=\frac{1}{\prod m_{i}^{\nu_{i}}} \Psi\left(\left\{c_{j l}\right\}\right)
$$

where $c_{j l}$ are defined in eq. (2.4).

Simplification of the integrals $J^{(N)}\left(n ; \nu_{1}, \ldots, \nu_{N}\right)$ when $\sum \nu_{i}=n$ is not surprising; to some extent, this may be considered a generalization of the so-called "uniqueness" formula for massless triangle diagrams [30] to the case of massive $N$-point integrals.

Whenever $\sum \nu_{i}=n$ the denominator of the integrand of (3.8) disappears, and the result is

$$
\left.J^{(N)}\left(n ; \nu_{1}, \ldots, \nu_{N}\right)\right|_{\Sigma \nu_{i}=n}=2 \mathrm{i}^{1-2 \Sigma \nu_{i}} \pi^{n / 2} \frac{\Gamma\left(\frac{n}{2}\right)}{\prod \Gamma\left(\nu_{i}\right)} \frac{1}{\prod m_{i}^{\nu_{i}}} \int_{0}^{\infty} \ldots \int_{0}^{\infty} \prod \alpha_{i}^{\nu_{i}-1} \mathrm{~d} \alpha_{i} \delta\left(\alpha^{T}\|c\| \alpha-1\right) .
$$

In particular, when all $\nu_{i}=1(n=N)$, our integrand is just a $\delta$ function,

$$
J^{(N)}(N ; 1, \ldots, 1)=2 \mathrm{i}^{1-2 N} \pi^{N / 2} \frac{\Gamma\left(\frac{N}{2}\right)}{\prod m_{i}} \int_{0}^{\infty} \ldots \int_{0}^{\infty} \prod \mathrm{d} \alpha_{i} \delta\left(\alpha^{T}\|c\| \alpha-1\right) .
$$

Consider also a very special situation, when all non-diagonal $c_{j l}$ vanish (i.e. $\left.c_{j l}=\delta_{j l}\right)$. Physically, this corresponds to the case where all external momenta squared take the mean values between the threshold and the pseudo-threshold, $k_{j l}^{2}=m_{j}^{2}+m_{l}^{2}$ (for $j<l$ ). In this case, all the angles $\tau_{j l}(j \neq l)$ are equal to $\frac{1}{2} \pi$. Employing the representation (3.12) we arrive at a very simple result for this special case,

$$
\left.J^{(N)}\left(n ; \nu_{1}, \ldots, \nu_{N}\right)\right|_{\substack{\Sigma \nu_{i}=n \\ c_{j l}=\delta_{j l}}}=\mathrm{i}^{1-2 \Sigma \nu_{i}} \pi^{n / 2} \frac{\prod \Gamma\left(\frac{1}{2} \nu_{i}\right)}{2^{N-1} \prod \Gamma\left(\nu_{i}\right)} \frac{1}{\prod m_{i}^{\nu_{i}}} .
$$

Imagine the $N$-dimensional Euclidean space of the parameters $\alpha_{i}$. The quadratic argument of the $\delta$ function in (3.13) defines a $(N-1)$-dimensional hypersurface. Provided that all eigenvalues of this quadratic form are positive, this is an $N$-dimensional ellipsoid. Otherwise, this is an $N$-dimensional hyperboloid. So, the integral (3.13) is nothing but the measure (i.e. the content) of the part of this hypersurface which belongs to the region 
where all $\alpha$ 's are non-negative. This region is a $2^{N}$-th part of the whole $N$-dimensional space.

To calculate the content of this part of the hypersurface, it is reasonable to diagonalize the quadratic form. Suppose there is an $N$-dimensional rotation transforming the old coordinates $\alpha_{i}$ into the new ones, $\beta_{i}$, so that

$$
\alpha^{T}\|c\| \alpha=\sum \alpha_{i}^{2}+2 \sum_{j<l} \alpha_{j} \alpha_{l} c_{j l} \Rightarrow \sum \lambda_{i} \beta_{i}^{2}
$$

Obviously, the product of $\lambda$ 's must be equal to the determinant $D^{(N)}$ of the matrix (2.13) corresponding to the quadratic form (3.6),

$$
\lambda_{1} \ldots \lambda_{N}=D^{(N)} .
$$

Note that this determinant $D^{(N)}$ is related to the content $V^{(N)}$ of the basic $N$-dimensional simplex via eq. (2.16).

Now, let us assume that all eigenvalues $\lambda_{i}$ are real and positive, i.e. the hyper-surface defined by the quadratic form is an $N$-dimensional ellipsoid. After rotation from $\alpha$ 's to $\beta$ 's, we get into the co-ordinate system corresponding to the principal axes of the ellipsoid. Then, we can rescale

$$
\beta_{i}=\frac{\gamma_{i}}{\sqrt{\lambda_{i}}}
$$

the Jacobian of this transformation being $\left(\lambda_{1} \ldots \lambda_{N}\right)^{-1 / 2}=\left(D^{(N)}\right)^{-1 / 2}$, according to (3.16). In this way, our $N$-dimensional ellipsoid is transformed into a hypersphere. Now all we need to calculate is the content of a part of this hypersphere which is cut out (in the space of $\gamma_{i}$ ) by the images of the hyperfaces restricting the region where all $\alpha_{i}$ are positive (in the space of $\alpha_{i}$ ). This content, which we shall denote by $\Omega^{(N)}$, can be understood as the $N$-dimensional solid angle subtended by the above-mentioned hyperfaces. In terms of $\Omega^{(N)}$, the relevant integral 3.13$)$ can be written as

$$
J^{(N)}(N ; 1, \ldots, 1)=\mathrm{i}^{1-2 N} \pi^{N / 2} \frac{\Gamma\left(\frac{N}{2}\right)}{\prod m_{i}} \frac{\Omega^{(N)}}{\sqrt{D^{(N)}}} .
$$

An interesting fact is that $\Omega^{(N)}$ is also related to the basic simplex. To see this, let us note that effectively (in terms of $\alpha$ 's), the transformation $\alpha_{i} \rightarrow \beta_{i} \rightarrow \gamma_{i}$ is equivalent to using the matrix $\|c\|$ as a new metric. Say, for a vector $\alpha$ the new length squared is

$$
|\alpha|_{c}^{2} \equiv \alpha^{T}\|c\| \alpha
$$

For unit vectors $e_{i}^{(\alpha)}$, the new scalar product is

$$
\left(e_{j}^{(\alpha)} \cdot e_{l}^{(\alpha)}\right)_{c}=c_{j l} \equiv \cos \tau_{j l} .
$$

Taking into account that $\left|e_{i}^{(\alpha)}\right|_{c}^{2}=1$, we see that in the space with the $c$-metric (i.e. in the space of $\gamma_{i}$ ) the angle between $e_{j}^{(\alpha)}$ and $e_{l}^{(\alpha)}$ is $\tau_{j l}$. Therefore, we get nothing but the $N$-dimensional solid angle at the mass meeting point of the basic simplex. It defines 
the region of integration on the hypersurface of the unit hypersphere in the $\gamma_{i}$ coordinate system. We also note that the cosines $c_{j l}$ are invariant under simple rescaling of $\alpha$ 's.

It is easy to see that the image of $e_{i}^{(\alpha)}$ in the $\gamma_{i}$-space is directed along the vector $a_{i}$. Moreover, according to eq. (2.34), the image of the vector with the components $F_{i}^{(N)} / m_{i}$ is directed along the height $H_{0}$.

To summarize, the following statement is valid: The content of the $N$-dimensional solid angle $\Omega^{(N)}$ in the space of $\gamma_{i}$ is equal to that at the vertex of the basic $N$-dimensional simplex where all mass sides meet. Moreover, the angles between the corresponding hyperfaces in the space of $\gamma_{i}$ and those in the basic simplex are the same. Therefore, the result for the integral (3.13) can be expressed in terms of the content of the basic simplex and the content of its $N$-dimensional solid angle at the vertex which is common for all mass sides as

$$
J^{(N)}(N ; 1, \ldots, 1)=\mathrm{i}^{1-2 N} \pi^{N / 2} \frac{\Gamma\left(\frac{N}{2}\right)}{N !} \frac{\Omega^{(N)}}{V^{(N)}} .
$$

Looking at eq. (3.21), we see that $\Omega^{(N)}$ is indeed the only thing which is to be calculated, since $V^{(N)}$ is known through eq. (2.16). In the following sections, we shall consider the lowest examples to understand how to calculate $\Omega^{(N)}$.

Nevertheless, the above discussion leads us to the following significant statement: The content $\Omega^{(N)}$ is nothing but the content of a non-Euclidean $(N-1)$-dimensional simplex calculated in the spherical (or hyperbolic, depending on the signature of the eigenvalues $\lambda_{i}$ ) space of constant curvature. The sides of this non-Euclidean simplex are equal to the angles $\tau_{j l}$. Therefore, the problem of calculating Feynman integrals is intimately connected with the problem of calculating the content of a simplex in non-Euclidean geometry.

\subsection{The general case}

To understand how to deal with the general case, when $\Sigma \nu_{i} \neq n$, we need some modification of the above transformations. First of all, we note that eqs. (2.33) can be rephrased as

$$
\sum_{l=1}^{N}\left(\sqrt{F_{j}^{(N)}} c_{j l} \sqrt{F_{l}^{(N)}}\right) \frac{\sqrt{F_{l}^{(N)}}}{m_{l}}=D^{(N)} \frac{\sqrt{F_{j}^{(N)}}}{m_{j}} .
$$

In other words, the vector with components

$$
f_{i}=\frac{\sqrt{F_{i}^{(N)}}}{m_{i}}
$$

is an eigenvector of the matrix $\|C\| \equiv\left\|C_{j l}\right\|$ with the elements

$$
C_{j l}=\left(\sqrt{F_{j}^{(N)}} c_{j l} \sqrt{F_{l}^{(N)}}\right)
$$

and the corresponding eigenvalue is equal to $D^{(N)}$. Note that

$$
f^{2} \equiv(f \cdot f)=\sum_{i=1}^{N} \frac{F_{i}^{(N)}}{m_{i}^{2}}=\frac{\Lambda^{(N)}}{\prod_{i=1}^{N} m_{i}^{2}},
$$




$$
\operatorname{det}\|C\|=D^{(N)} \prod_{j=1}^{N} F_{j}^{(N)} .
$$

The idea is that it may be more convenient to use the quadratic form defined by the matrix $\left\|C_{j l}\right\|$, rather than $\left\|c_{j l}\right\|$, since we know one of its eigenvectors with the corresponding eigenvalue.

After using the masses $m_{i}$ to rescale the Feynman parameters $\alpha_{i}$ and "hiding" the quadratic form into the argument of the delta function, we obtained the representation (3.8). Analogously, we can use $1 / f_{i}=m_{i} / \sqrt{F_{i}^{(N)}}$, rather than $m_{i}$, to rescale the original $\alpha$ 's. The result is

$$
\begin{aligned}
& J^{(N)}\left(n ; \nu_{1}, \ldots, \nu_{N}\right) \\
& \quad=2 \mathrm{i}^{1-2 \Sigma \nu_{i}} \pi^{n / 2} \frac{\Gamma\left(\sum \nu_{i}-\frac{n}{2}\right)}{\prod \Gamma\left(\nu_{i}\right)}\left(\prod f_{i}^{\nu_{i}}\right) \int_{0}^{\infty} \ldots \int_{0}^{\infty} \frac{\prod \alpha_{i}^{\nu_{i}-1} \mathrm{~d} \alpha_{i}}{\left(\sum \alpha_{i} f_{i}\right)^{n-\Sigma \nu_{i}}} \delta\left(\alpha^{T}\|C\| \alpha-1\right),
\end{aligned}
$$

where notation (3.6) has been used again. A nice feature of this representation is that in the denominator we have got the contraction (scalar product) of $\alpha$ and the known eigenvector of the matrix $\|C\|$ defining the quadratic form (see eqs. (3.22) $-(3.24)$ ).

If $\nu_{1}=\ldots=\nu_{N}=1$, we get

$$
J^{(N)}(n ; 1, \ldots, 1)=2 \mathrm{i}^{1-2 N} \pi^{n / 2} \Gamma\left(N-\frac{n}{2}\right)\left(\prod f_{i}\right) \int_{0}^{\infty} \ldots \int_{0}^{\infty} \frac{\prod \mathrm{d} \alpha_{i}}{\left(\sum \alpha_{i} f_{i}\right)^{n-N}} \delta\left(\alpha^{T}\|C\| \alpha-1\right) .
$$

Now, let us use the same transformations of variables as earlier. Namely, let us first rotate from $\alpha_{i}$ to the variables $\beta_{i}$ such that the quadratic form becomes diagonal,

$$
\alpha^{T}\|C\| \alpha=\sum_{i=1}^{N} \lambda_{i} \beta_{i}^{2}
$$

One of these $\beta$ 's, say $\beta_{N}$, is directed along the eigenvector $f$. Therefore, $\lambda_{N}=D^{(N)}$ and $\prod_{i=1}^{N-1} \lambda_{i}=\prod_{j=1}^{N} F_{j}^{(N)}$. Moreover, the denominator is also proportional to $\beta_{N}$. Finally, we rescale $\beta_{i}=\gamma_{i} / \sqrt{\lambda_{i}}$, and the denominator becomes

$$
\left(\sum \alpha_{i} f_{i}\right) \Rightarrow \frac{1}{\prod m_{i}} \sqrt{\frac{\Lambda^{(N)}}{D^{(N)}}} \gamma_{N}=\frac{1}{m_{0}} \gamma_{N}
$$

where $m_{0}$, defined in eq. (2.21), is the distance between the mass meeting point and the momentum hyperface (i.e. the length of the height $H_{0}$ ).

Now, the transformation $\alpha_{i} \rightarrow \beta_{i} \rightarrow \gamma_{i}$ is equivalent to using the matrix $\|C\|$ as a new metric. For a vector $\alpha$ the new length squared is

$$
|\alpha|_{C}^{2} \equiv \alpha^{T}\|C\| \alpha
$$

For unit vectors $e_{i}^{(\alpha)}$, the new scalar product is

$$
\left(e_{j}^{(\alpha)} \cdot e_{l}^{(\alpha)}\right)_{C}=C_{j l},
$$


and the corresponding cosine of the angle is

$$
\frac{\left(e_{j}^{(\alpha)} \cdot e_{l}^{(\alpha)}\right)_{C}}{\left|e_{j}^{(\alpha)}\right|_{C}\left|e_{l}^{(\alpha)}\right|_{C}}=\frac{C_{j l}}{\sqrt{C_{j j} C_{l l}}}=c_{j l} \equiv \cos \tau_{j l}
$$

Again, as in the case of the $c$-metric, we get the same $N$-dimensional solid angle as that occurring at the mass meeting point of the basic simplex.

But we can go further. In particular, we can comprehend what is the image of the vector $f$. Consider the scalar products

$$
\begin{gathered}
\left(e_{i}^{(\alpha)} \cdot f\right)_{C}=\left(e_{i}^{(\alpha)} \cdot\|C\| f\right)=D^{(N)}\left(e_{i}^{(\alpha)} \cdot f\right)=D^{(N)} f_{i}, \\
(f \cdot f)_{C}=(f \cdot\|C\| f)=D^{(N)}(f \cdot f)=\frac{D^{(N)} \Lambda^{(N)}}{\prod_{j=1}^{N} m_{j}^{2}} .
\end{gathered}
$$

Hence, the cosine of the angle between the images of $f$ and $e_{i}^{(\alpha)}$ is (cf. eq. (2.44))

$$
\frac{\left(e_{i}^{(\alpha)} \cdot f\right)_{C}}{\sqrt{\left(e_{i}^{(\alpha)} \cdot e_{i}^{(\alpha)}\right)_{C}(f \cdot f)_{C}}}=\sqrt{\frac{D^{(N)}}{\Lambda^{(N)}}} \prod_{j \neq i} m_{j}=\frac{m_{0}}{m_{i}}=\cos \tau_{0 i} \equiv c_{0 i} .
$$

Therefore, in the basic simplex, with the mass sides $m_{i}$, etc., the image of the vector $f$ is directed along the height $H_{0}$ !

In terms of $\gamma$ 's, the integral becomes

$$
J^{(N)}(n ; 1, \ldots, 1)=2 \mathrm{i}^{1-2 N} \pi^{n / 2} \Gamma\left(N-\frac{n}{2}\right) \frac{m_{0}^{n-N-1}}{\sqrt{\Lambda^{(N)}}} \underset{\Omega^{(N)}}{\int \ldots} \frac{\prod \mathrm{d} \gamma_{i}}{\gamma_{N}^{n-N}} \delta\left(\sum \gamma_{i}^{2}-1\right)
$$

where the integration goes over the interior of the $N$-dimensional solid angle $\Omega^{(N)}$ of the basic simplex. If we define the angle between the "running" unit vector and the $N$-th axis as $\theta$, the above formula means that we should integrate over the hypersurface of the unit hypersphere with the "weight" $(\cos \theta)^{N-n}$, within the limits set by $\Omega^{(N)}$,

$$
J^{(N)}(n ; 1, \ldots, 1)=\mathrm{i}^{1-2 N} \pi^{n / 2} \Gamma\left(N-\frac{n}{2}\right) \frac{m_{0}^{n-N}}{\sqrt{D^{(N)}} \prod m_{i}} \Omega^{(N ; n)},
$$

with

$$
\Omega^{(N ; n)} \equiv \int \underset{\Omega^{(N)}}{\int} \frac{\mathrm{d} \Omega_{N}}{\cos ^{n-N} \theta}
$$

Obviously, $\Omega^{(N ; N)}=\Omega^{(N)}$. Using eq. (2.16), the formula (3.38) can be exhibited in the following form

$$
J^{(N)}(n ; 1, \ldots, 1)=\mathrm{i}^{1-2 N} \pi^{n / 2} \Gamma\left(N-\frac{n}{2}\right) \frac{m_{0}^{n-N} \Omega^{(N ; n)}}{N ! V^{(N)}} .
$$

\footnotetext{
${ }^{12}$ Note that in the $\gamma_{i}$-space with the $C$-metric the $e_{i}^{(\alpha)}$ vectors are not unit, $\left|e_{i}^{(\alpha)}\right|_{C}^{2}=C_{i i}=F_{i}^{(N)}$.
} 


\subsection{Splitting the $N$-dimensional solid angle}

Now, let us again use the height $H_{0}$ to split the basic $N$-dimensional simplex into $N$ rectangular ones, each time replacing one of the mass sides, $m_{i}$, by $m_{0}=\left|H_{0}\right|$. The

content of the $i$-th rectangular simplex $V_{i}^{(N)}$ is given by eq. (2.41). Let us denote the corresponding $N$-dimensional solid angle as $\Omega_{i}^{(N)}$, so that

$$
\Omega_{i}^{(N ; n)}=\int \underset{\Omega_{i}^{(N)}}{\int \ldots} \frac{\mathrm{d} \Omega_{N}}{\cos ^{n-N} \theta}, \quad \Omega^{(N ; n)}=\sum_{i=1}^{N} \Omega_{i}^{(N ; n)} .
$$

Substituting (3.41) into eq. (3.40), we get

$$
J^{(N)}(n ; 1, \ldots, 1)=\sum_{i=1}^{N} \frac{V_{i}^{(N)}}{V^{(N)}} J_{i}^{(N)}(n ; 1, \ldots, 1),
$$

where $J_{i}^{(N)}$ are the integrals corresponding to the rectangular simplices. Specifically, in the integral $J_{i}^{(N)}$ the internal masses are

$$
m_{1}, \ldots, m_{i-1}, m_{0}, m_{i+1}, \ldots, m_{N}
$$

while the momenta invariants are

$$
\left\{\begin{array}{l}
k_{j l}^{2}, \quad \text { if } j \neq i \text { and } l \neq i \\
m_{l}^{2}-m_{0}^{2}, \quad \text { if } j=i \\
m_{j}^{2}-m_{0}^{2}, \quad \text { if } l=i
\end{array} .\right.
$$

Using (2.41), we arrive at

$$
J^{(N)}(n ; 1, \ldots, 1)=\frac{1}{\Lambda^{(N)}}\left(\prod m_{i}^{2}\right) \sum_{i=1}^{N} \frac{1}{m_{i}^{2}} F_{i}^{(N)} J_{i}^{(N)}(n ; 1, \ldots, 1) .
$$

We are now ready to tackle some specific examples using the geometrical approach.

\section{Two-point function}

Here, the two-dimensional basic simplex is a triangle and the angle $\tau_{12}$ lies between the two mass sides (see Fig. 4). We obtain

$$
\begin{gathered}
D^{(2)}=1-c_{12}^{2}=\sin ^{2} \tau_{12}, \quad V^{(2)}=\frac{1}{2} m_{1} m_{2} \sin \tau_{12}, \quad \Omega^{(2)}=\tau_{12}, \\
m_{0}=m_{1} m_{2} \sqrt{\frac{D^{(2)}}{\Lambda^{(2)}}}, \quad \cos \tau_{0 i}=\frac{m_{0}}{m_{i}}, \quad \tau_{01}+\tau_{02}=\tau_{12}, \quad \Lambda^{(2)}=k_{12}^{2} .
\end{gathered}
$$

In two dimensions, from (3.21) we obtain the well-known result

$$
J^{(2)}(2 ; 1,1)=\frac{\mathrm{i} \pi}{m_{1} m_{2}} \frac{\tau_{12}}{\sin \tau_{12}},
$$


while in three dimensions, eqs. (3.40)-(3.41) yield

$$
J^{(2)}(3 ; 1,1)=\mathrm{i} \pi^{2} \frac{1}{\sqrt{\Lambda^{(2)}}}\left\{\Omega_{1}^{(2 ; 3)}+\Omega_{2}^{(2 ; 3)}\right\}
$$

with

$$
\Omega_{i}^{(2 ; 3)}=\int_{0}^{\tau_{0 i}} \frac{\mathrm{d} \theta}{\cos \theta}=\ln \left(\frac{1+\sin \tau_{0 i}}{1-\sin \tau_{0 i}}\right) .
$$

Combining the logarithms, we get

$$
J^{(2)}(3 ; 1,1)=\frac{\mathrm{i} \pi^{2}}{\sqrt{k_{12}^{2}}} \ln \left(\frac{m_{1}+m_{2}+\sqrt{k_{12}^{2}}}{m_{1}+m_{2}-\sqrt{k_{12}^{2}}}\right) .
$$

In the Euclidean region $\left(k_{12}^{2}<0\right)$ the logarithm gives $\arctan \left(\sqrt{-k_{12}^{2}} /\left(m_{1}+m_{2}\right)\right)$, and the result coincides with those presented in [11, 31.

In four dimensions, the $\Gamma$ function in front of the integral (3.38) becomes singular. Introducing dimensional regularization [32] to circumvent that difficulty, we get

$$
J^{(2)}(4-2 \varepsilon ; 1,1)=\mathrm{i} \pi^{2-\varepsilon} \Gamma(\varepsilon) \frac{m_{0}^{1-2 \varepsilon}}{\sqrt{\Lambda^{(2)}}}\left\{\Omega_{1}^{(2 ; 4-2 \varepsilon)}+\Omega_{2}^{(2 ; 4-2 \varepsilon)}\right\},
$$

with

$$
\Omega_{i}^{(2 ; 4-2 \varepsilon)}=\int_{0}^{\tau_{0 i}} \frac{\mathrm{d} \theta}{\cos ^{2-2 \varepsilon} \theta} .
$$

Expanding the integrand in $\varepsilon$ and taking into account that

$$
\int_{0}^{\tau} \frac{\mathrm{d} \theta}{\cos ^{2} \theta} \ln (\cos \theta)=\tan \tau \ln (\cos \tau)+\tan \tau-\tau,
$$

we reach the well-known result (cf., e.g., in [2, 33])

$$
\begin{aligned}
& J^{(2)}(4-2 \varepsilon ; 1,1)=\mathrm{i} \pi^{2-\varepsilon} \Gamma(1+\varepsilon) \\
\times & \left\{\frac{1}{\varepsilon}+2-\ln m_{1}-\ln m_{2}+\frac{m_{1}^{2}-m_{2}^{2}}{k_{12}^{2}} \ln \frac{m_{2}}{m_{1}}-\frac{2 m_{1} m_{2}}{k_{12}^{2}} \tau_{12} \sin \tau_{12}\right\}+\mathcal{O}(\varepsilon) .
\end{aligned}
$$

Furthermore, the representation (4.7)-(4.8) makes it possible to construct next terms of the expansion in $\varepsilon$. For example, the $\varepsilon$ term requires just ${ }^{3}$

$$
\int_{0}^{\tau} \frac{\mathrm{d} \theta}{\cos ^{2} \theta} \ln ^{2}(\cos \theta)=\tan \tau\left(\ln ^{2}(\cos \tau)+2 \ln (\cos \tau)+2\right)-2 \tau(1-\ln 2)-\mathrm{Cl}_{2}(\pi-2 \tau)
$$

\footnotetext{
${ }^{13}$ The Clausen function $\mathrm{Cl}_{2}(\theta)$ is defined in Appendix A.
} 
(the corresponding result was obtained in [34). Moreover, the result for an arbitrary space-time dimension or $\varepsilon$ can be obtained in terms of the Gauss hypergeometric function (cf. in 35, 33]), using

$$
\Omega_{i}^{(2 ; 4-2 \varepsilon)}=\int_{0}^{\tau_{0 i}} \frac{\mathrm{d} \theta}{\cos ^{2-2 \varepsilon} \theta}=2 \tan \tau_{0 i}{ }_{2} F_{1}\left(\begin{array}{c}
1 / 2, \varepsilon \\
3 / 2
\end{array} \mid-\tan ^{2} \tau_{0 i}\right) .
$$

Using formulae of analytic continuation of ${ }_{2} F_{1}$ function, one can establish a connection with the result presented in eq. (A.7) of [33].

\section{Three-point function}

\subsection{Geometrical picture and the three-dimensional case}

Here the three-dimensional basic simplex is a tetrahedron with three mass sides (the angles between these mass sides are $\tau_{12}, \tau_{13}$ and $\tau_{23}$ ) and three momentum sides. It is shown in Fig. 5a. The volume of this tetrahedron is defined as

$$
V^{(3)}=\frac{1}{6} m_{1} m_{2} m_{3} \sqrt{D^{(3)}}
$$

where (cf. eqs. (2.13)-(2.14))

$$
D^{(3)} \equiv\left|\begin{array}{ccc}
1 & c_{12} & c_{13} \\
c_{12} & 1 & c_{23} \\
c_{13} & c_{23} & 1
\end{array}\right|=1-c_{12}^{2}-c_{13}^{2}-c_{23}^{2}+2 c_{12} c_{13} c_{23} .
$$

Furthermore, $\Omega^{(3)}$ is the usual solid angle at the vertex derived by the mass sides (cf. Fig. $5 \mathrm{~b}$ ). Its value can be defined as the area of a part of the unit sphere cut out by the three planar faces adjacent to the vertex; in other words, this is the area of a spherical triangle corresponding to this section. The sides of this spherical triangle are obviously equal to the angles $\tau_{12}, \tau_{13}$ and $\tau_{23}$ while its angles, $\psi_{12}, \psi_{13}$ and $\psi_{23}$, are equal to those between the plane faces (we define $\psi_{12}$ as the angle between the sides $\tau_{13}$ and $\tau_{23}$, etc.). This definition is in agreement with eq. (2.27). Using eq. (2.24) or the well-known formulae of the spherical trigonometry, it is easy to show that

$$
\cos \psi_{12}=\frac{\cos \tau_{12}-\cos \tau_{13} \cos \tau_{23}}{\sin \tau_{13} \sin \tau_{23}}, \quad \sin \psi_{12}=\frac{\sqrt{D^{(3)}}}{\sin \tau_{13} \sin \tau_{23}},
$$

and analogous expressions for $\psi_{13}$ and $\psi_{23}$. The solid angle corresponding to spherical triangle can be obtained via

$$
\Omega^{(3)}=\psi_{12}+\psi_{13}+\psi_{23}-\pi
$$

Using eqs. (5.3) and (5.4), it is straightforward to show that

$$
\Omega^{(3)}=2 \arccos \left(\frac{1+c_{12}+c_{13}+c_{23}}{\sqrt{2\left(1+c_{12}\right)\left(1+c_{13}\right)\left(1+c_{23}\right)}}\right)
$$




$$
\begin{aligned}
& =2 \arcsin \left(\sqrt{\frac{D^{(3)}}{2\left(1+c_{12}\right)\left(1+c_{13}\right)\left(1+c_{23}\right)}}\right) \\
& =2 \arctan \left(\frac{\sqrt{D^{(3)}}}{1+c_{12}+c_{13}+c_{23}}\right) .
\end{aligned}
$$

Finally, the result

$$
J^{(3)}(3 ; 1,1,1)=-\frac{\mathrm{i} \pi^{2}}{2 m_{1} m_{2} m_{3}} \frac{\Omega^{(3)}}{\sqrt{D^{(3)}}},
$$

with $\Omega^{(3)}$ defined by (5.5), corresponds to one obtained in [11] in a different way. Note that here we have derived it purely geometrically.

However, eq. (5.4) cannot be easily generalized to the four-dimensional case. This is why it is instructive to reproduce the three-dimensional result (5.5) in a different manner, splitting the basic tetrahedron into rectangular ones (cf. eq. (3.42)).

Consider a spherical triangle 123 on the unit sphere, cut out by the solid angle of the basic tetrahedron in the $\gamma$ space, as illustrated in Fig. 6 . The points 1,2,3 correspond to the intersections of the mass sides of the basic tetrahedron and the unit sphere. The sides of the triangle are equal to the angles $\tau_{12}, \tau_{23}$ and $\tau_{31}$. There is another point 0 , also on the unit sphere, corresponding to the intersection of the height $H_{0}$ of the tetrahedron and the unit sphere. The point 0 is connected with each of the points $1,2,3$. The corresponding sides 01,02 and 03 are equal to $\tau_{01}, \tau_{02}$ and $\tau_{03}$, respectively, so that

$$
\cos \tau_{0 i}=\frac{m_{0}}{m_{i}}, \quad m_{0}=m_{1} m_{2} m_{3} \sqrt{\frac{D^{(3)}}{\Lambda^{(3)}}}
$$

with (cf. eq. (2.19))

$$
\Lambda^{(3)}=-\frac{1}{4}\left[\left(k_{12}^{2}\right)^{2}+\left(k_{13}^{2}\right)^{2}+\left(k_{23}^{2}\right)^{2}-2 k_{12}^{2} k_{13}^{2}-2 k_{12}^{2} k_{23}^{2}-2 k_{13}^{2} k_{23}^{2}\right]=-\frac{1}{4} \lambda\left(k_{12}^{2}, k_{13}^{2}, k_{23}^{2}\right),
$$

where $\lambda(x, y, z)$ is defined in eq. (1.1) (cf. Fig. 1). The triangle 123 is thereby split into three triangles 012, 023 and 031 . The angle between 01 and 02 is denoted as $\varphi_{12}$, etc. Obviously,

$$
\varphi_{12}+\varphi_{23}+\varphi_{31}=2 \pi \text {. }
$$

For the calculation of three-dimensional triangle integral, it does not really matter where the point 0 lies, though this becomes essential for the four-dimensional case. As we know, the $N=n$ integrals can be reduced to the integrals with equal masses. So, let us start with the equal mass case. In Fig. 6 , this means that $\tau_{01}=\tau_{02}=\tau_{03} \equiv \tau_{0}$. Therefore, the spherical triangles 012, 023 and 031 are isosceles. Consider one of these triangles, say 012, as illustrated in Fig. 7. The sides 01 and 02 are equal to $\tau_{0}$. The third side, 12, is equal to $\tau_{12}$. The length of the perpendicular dropped from the point 0 to the side 12 is denoted as $\eta_{12}$. This perpendicular intersects the side 12 in a point $T_{12}$ which in the equal-mass case divides the side 12 into two equal parts (each of them equals $\tau_{12} / 2$ ). It also divides the angle $\varphi_{12}$ into two equal parts. The angles at the vertices 1 and 2 (which are also equal in the equal-mass case) are denoted as $\kappa_{12}$. The lengths of two of the sides of a smaller isosceles triangle $01_{\xi} 2_{\xi}, 01_{\xi}$ and $02_{\xi}$, are $\tau_{0} \xi(0 \leq \xi \leq 1)$. The third side, 
$1_{\xi} 2_{\xi}$, is parallel (in the spherical sense) to the side 12 and its length is denoted as $\tau(\xi)$; obviously, $\tau(0)=0$ and $\tau(1)=\tau_{12}$. The perpendicular $0 T_{12}$ also splits it into two equal parts of the length $\tau(\xi) / 2$. The length of the part of the perpendicular within the triangle $01_{\xi} 2_{\xi}$ is denoted as $\eta(\xi)$, whereas the angles at the vertices $1_{\xi}$ and $2_{\xi}$ are $\kappa(\xi)$; obviously, $\eta(0)=0, \eta(1)=\eta_{12}, \kappa(0)=\left(\pi-\varphi_{12}\right) / 2, \kappa(1)=\kappa_{12}$.

The main idea of introducing an auxiliary variable $\xi$ is to simplify the limits of integration. We define the usual spherical integration angle $\theta$ in a way that it is the angle between the direction of $H_{0}$ and the "running" point. For the triangle 012 presented in Fig. 7, let us define that $\varphi=0$ corresponds to the direction of the perpendicular $0 T_{12}$. In this way, the limits of the $\varphi$ integration are $\pm \varphi_{12} / 2$. The lower limit of the $\theta$ integration is zero, whereas the upper limit depends on $\varphi$ and varies from $\tau_{0}$ (at $\varphi= \pm \varphi_{12} / 2$ ) to $\eta_{12}$ (at $\left.\varphi=0\right)$. If we use, instead of $\theta$, the variable $\xi$, its limits will be $0 \leq \xi \leq 1$, independently of $\varphi$. The result of an infinitesimal variation of $\xi$ will be a thin strip based on the side $1_{\xi} 2_{\xi}$ (cf. Fig. 7). Moreover, all such strips from the triangles 012, 023 and 031 (cf. Fig. 6) can be "glued" together. So, the whole result of a variation in $\xi$ is a thin closed spherical triangle. Alternatively, one can perform the azimuthal $\varphi$-integration first, and then evaluate the remaining $\xi$ integral. As we shall see, both ways work in the three-point case.

Using formulae of spherical trigonometry (cf. Fig. 7), we get the following relations:

$$
\begin{aligned}
\sin \left(\frac{1}{2} \tau(\xi)\right) & =\sin \left(\frac{1}{2} \varphi_{12}\right) \sin \left(\tau_{0} \xi\right) \\
\sin \eta(\xi) & =\sin \kappa(\xi) \sin \left(\tau_{0} \xi\right) \\
\cos \kappa(\xi) & =\sin \left(\frac{1}{2} \varphi_{12}\right) \cos \eta(\xi) \\
\tan \kappa(\xi) & =\left(\cos \left(\tau_{0} \xi\right) \tan \left(\frac{1}{2} \varphi_{12}\right)\right)^{-1} \\
\tan \eta(\xi) & =\cos \left(\frac{1}{2} \varphi_{12}\right) \tan \left(\tau_{0} \xi\right)
\end{aligned}
$$

The situation for arbitrary $\varphi$ is drawn in Fig. 8. This is a rectangular spherical triangle with an upper vertex 0 and one of the outgoing sides being the perpendicular of the length $\eta(\xi)$, as in Fig. 7. This side corresponds to $\varphi=0$. The angle at the vertex 0 is equal to $\varphi$ and a running value of $\varphi$ is understood. The side opposite to 0 is perpendicular to the one mentioned before: $\eta(\xi)$. This is a part of the corresponding side directed to the point $1_{\xi}$ in Fig. 7, i.e. the line of constant $\xi$. Its length is denoted as $\rho(\xi, \varphi)$. The third side or "hypotenuse" has length $\theta(\xi, \varphi)$. The vertex opposite to the $\eta(\xi)$ side is the running point with the coordinates $\theta, \varphi$. The angle at this vertex is denoted as $\kappa(\xi, \varphi)$.

We want to express $\theta$ in terms of $\xi$ and $\varphi$. Using the formulae of spherical trigonometry for the triangle in Fig. 8, analogous to ones written above, it is straightforward to show that

$$
\tan \theta(\xi, \varphi)=\frac{\tan \eta(\xi)}{\cos \varphi}=\frac{\cos \left(\frac{1}{2} \varphi_{12}\right)}{\cos \varphi} \tan \left(\tau_{0} \xi\right)
$$

The area is obtained by integrating

$$
\sin \theta d \theta d \varphi=-d(\cos \theta) d \varphi \Rightarrow-\frac{\partial(\cos \theta(\xi, \varphi))}{\partial \xi} d \xi d \varphi,
$$


where, according to eq. (5.10),

$$
\cos \theta(\xi, \varphi)=\left(1+\frac{\cos ^{2}\left(\frac{1}{2} \varphi_{12}\right)}{\cos ^{2} \varphi} \tan ^{2}\left(\tau_{0} \xi\right)\right)^{-1 / 2}
$$

If we first perform the $\varphi$-integral, this effectively corresponds to the area of the infinitesimal strip between two sides of constant $\xi$,

$$
-2 \mathrm{~d}\left\{\arctan \left(\cos \left(\tau_{0} \xi\right) \tan \left(\frac{1}{2} \varphi_{12}\right)\right)\right\} \text {. }
$$

Then, the remaining $\xi$ integral gives just this arctan. Collecting the results for all triangles (cf. Fig. 6), we get the same as in eq. (5.5). This can be seen if we observe that the expression (5.13) is nothing but $2 \mathrm{~d} \kappa(\xi)$ and the spherical excess for the triangle $01_{\xi} 2_{\xi}$ is $\left(\varphi_{12}+2 \kappa(\xi)-\pi\right)$. A nice thing about eq. (5.13) is that in the $\xi$-integral we can glue all arctan functions into a symmetric answer at the infinitesimal level. The same final result can also be obtained when we first integrate over $\xi$, it being trivial since the integrand is a derivative with respect to $\xi$.

\subsection{The four-dimensional case}

If we consider the four-dimensional three-point function, the only (but very essential!) difference is that we should divide the integrand by $\cos \theta(\xi, \varphi)$, cf. eq. (3.37). Therefore, the function in the integrand can be written as

$$
-\frac{\partial}{\partial \xi} \ln (\cos \theta(\xi, \varphi))
$$

As in the previous case, one can integrate first over either $\varphi$ or $\xi$; both ways lead to equivalent results. Let us integrate first over $\xi$, this gives

$$
\ln \left(\frac{\cos \theta(0, \varphi)}{\cos \theta(1, \varphi)}\right)=\frac{1}{2} \ln \left(1+\frac{\tan ^{2} \eta_{12}}{\cos ^{2} \varphi}\right)
$$

In the equal-mass case, the remaining $\varphi$-integral becomes

$$
\begin{aligned}
& \int_{0}^{\varphi_{12} / 2} \mathrm{~d} \varphi \ln \left(1+\frac{\tan ^{2} \eta_{12}}{\cos ^{2} \varphi}\right) \\
& =\frac{1}{2} \int_{0}^{\varphi_{12}} \mathrm{~d} \varphi^{\prime}\left\{\ln \left(1+2 \tan ^{2} \eta_{12}\right)+\ln \left(1+\frac{\cos \varphi^{\prime}}{1+2 \tan ^{2} \eta_{12}}\right)-\ln \left(1+\cos \varphi^{\prime}\right)\right\}
\end{aligned}
$$

where $\varphi^{\prime}=2 \varphi$. Using a result from [27] (p. 308, eq. (38)), this integral can be expressed in terms of the Clausen function (see in Appendix A) as

$$
\frac{1}{2} \tau_{12} \ln \left(\frac{\sin \left(\frac{1}{2}\left(\varphi_{12}+\tau_{12}\right)\right)}{\sin \left(\frac{1}{2}\left(\varphi_{12}-\tau_{12}\right)\right)}\right)+\frac{1}{2} \mathrm{Cl}_{2}\left(\varphi_{12}+\tau_{12}\right)+\frac{1}{2} \mathrm{Cl}_{2}\left(\varphi_{12}-\tau_{12}\right)-\mathrm{Cl}_{2}\left(\varphi_{12}\right)
$$


The arguments of the Clausen functions have a very transparent geometric interpretation. We note that the logarithmic term can also be presented as

$$
\frac{1}{2} \tau_{12} \ln \left(\frac{1+\sin \eta_{12}}{1-\sin \eta_{12}}\right) .
$$

The function (5.17) can also be expressed in terms of the generalized inverse tangent integral (A.8) as

$$
\mathrm{Ti}_{2}\left(\tan \left(\frac{1}{2} \tau_{12}\right), \tan \left(\frac{1}{2} \varphi_{12}\right)\right)-\mathrm{Ti}_{2}\left(\tan \left(\frac{1}{2} \tau_{12}\right),-\tan \left(\frac{1}{2} \varphi_{12}\right)\right) .
$$

The generalization to the case of unequal masses is easy. To understand why, let us consider Fig. 9. This is again a close-up of the triangle 012 from Fig. 6, but now it is asymmetric, because $\tau_{01}$ and $\tau_{02}$ are different. We denote the quantities in one of the triangles as $\varphi_{12}^{+} / 2, \tau_{12}^{+} / 2, \kappa_{12}^{+} / 2$, and in another triangle as $\varphi_{12}^{-} / 2, \tau_{12}^{-} / 2, \kappa_{12}^{-} / 2$. The height $\eta_{12}$ is the same for both triangles. Obviously,

$$
\frac{1}{2}\left(\varphi_{12}^{+}+\varphi_{12}^{-}\right)=\varphi_{12} \quad \text { and } \quad \frac{1}{2}\left(\tau_{12}^{+}+\tau_{12}^{-}\right)=\tau_{12}
$$

One can immediately see that the result for the integral over this asymmetric triangle is nothing but a half of the sum of the functions (5.17) labelled with plus and with minus. Namely,

$$
\begin{aligned}
\frac{1}{2} \tau_{12} \ln \left(\frac{1+\sin \eta_{12}}{1-\sin \eta_{12}}\right) & +\frac{1}{4} \mathrm{Cl}_{2}\left(\varphi_{12}^{+}+\tau_{12}^{+}\right)+\frac{1}{4} \mathrm{Cl}_{2}\left(\varphi_{12}^{+}-\tau_{12}^{+}\right)-\frac{1}{2} \mathrm{Cl}_{2}\left(\varphi_{12}^{+}\right) \\
& +\frac{1}{4} \mathrm{Cl}_{2}\left(\varphi_{12}^{-}+\tau_{12}^{-}\right)+\frac{1}{4} \mathrm{Cl}_{2}\left(\varphi_{12}^{-}-\tau_{12}^{-}\right)-\frac{1}{2} \mathrm{Cl}_{2}\left(\varphi_{12}^{-}\right)
\end{aligned}
$$

Again, the geometric interpretation of the arguments of the Clausen functions is very transparent, thanks to Fig. 9.

Some useful relations are

$$
\begin{array}{clrl}
\cos \left(\frac{1}{2} \tau_{12}^{+}\right)=\frac{\cos \tau_{01}}{\cos \eta_{12}}, & \cos \left(\frac{1}{2} \tau_{12}^{-}\right) & =\frac{\cos \tau_{02}}{\cos \eta_{12}}, \\
\sin \left(\frac{1}{2} \tau_{12}^{+}\right)=\sin \tau_{01} \sin \left(\frac{1}{2} \varphi_{12}^{+}\right), & \sin \left(\frac{1}{2} \tau_{12}^{-}\right)=\sin \tau_{02} \sin \left(\frac{1}{2} \varphi_{12}^{-}\right), \\
\tan \left(\frac{1}{2} \tau_{12}^{+}\right)=\sin \eta_{12} \tan \left(\frac{1}{2} \varphi_{12}^{+}\right), & \tan \left(\frac{1}{2} \tau_{12}^{-}\right)=\sin \eta_{12} \tan \left(\frac{1}{2} \varphi_{12}^{-}\right) .
\end{array}
$$

Worth noting is

$$
\cos \eta_{12}=\frac{m_{0} \sqrt{k_{12}^{2}}}{m_{1} m_{2} \sin \tau_{12}} .
$$

To get the complete result for the three-point integral in four dimensions, we should sum up the functions (5.21) for all three triangles 012, 023 and 031, and multiply by the overall factor (see eq. (3.37)) which is

$$
-\mathrm{i} \pi^{2} \frac{1}{\sqrt{\Lambda^{(3)}}}
$$

Note that in this case we get $\sqrt{\Lambda^{(3)}}$ rather than $\sqrt{D^{(3)}}$ in the denominator. 


\section{Four-point function}

\subsection{The general case}

In this case, the four-dimensional simplex has four mass sides and six momentum sides (see in Fig. 3). It has five vertices and five three-dimensional hyperfaces. Four of these hyperfaces are the reduced ones, corresponding to three-point functions, whereas the fifth one is the momentum hyperface. In fact, this four-dimensional simplex is completely defined by its mass sides $m_{1}, m_{2}, m_{3}, m_{4}$ and six "planar" angles between them, $\tau_{12}, \tau_{13}, \tau_{14}, \tau_{23}, \tau_{24}$ and $\tau_{34}$. According to eq. (2.16), the content (hyper-volume) of this simplex equals

$$
V^{(4)}=\frac{1}{24} m_{1} m_{2} m_{3} m_{4} \sqrt{D^{(4)}},
$$

with $D^{(4)}$ given by (2.13)-(2.14) at $N=4$,

$$
D^{(4)}=\operatorname{det}\left\|c_{j l}\right\|, \quad\left\|c_{j l}\right\|=\left(\begin{array}{cccc}
1 & c_{12} & c_{13} & c_{14} \\
c_{12} & 1 & c_{23} & c_{24} \\
c_{13} & c_{23} & 1 & c_{34} \\
c_{14} & c_{24} & c_{34} & 1
\end{array}\right) .
$$

The four-dimensional four-point function can be exhibited as (cf. eq. (3.18), (3.21)):

$$
J^{(4)}(4 ; 1,1,1,1)=\frac{1}{12} \mathrm{i} \pi^{2} \frac{\Omega^{(4)}}{V^{(4)}}=\frac{2 \mathrm{i} \pi^{2}}{m_{1} m_{2} m_{3} m_{4}} \frac{\Omega^{(4)}}{\sqrt{D^{(4)}}} .
$$

So, the main problem is how to calculate $\Omega^{(4)}$.

In four dimensions, $\Omega^{(4)}$ is the value of the four-dimensional generalization of the solid angle at the vertex of the simplex where all four mass sides meet. In the spherical case, it can be defined as the volume of a part of the unit hypersphere which is cut out from it by the four three-dimensional reduced hyperfaces, each hyperface involving three mass sides of the simplex. This hyper-section is a three-dimensional spherical tetrahedron $\square^{\mathbb{4}}$ whose six sides (edges) are equal to the angles $\tau_{j l}$.

It is illustrated in Fig. 10 where the $i$-th vertex $(i=1,2,3,4)$ corresponds to the intersection of the $i$-th mass side of the basic simplex and the unit hyperspheret. Furthermore, the dihedral angles of this three-dimensional spherical tetrahedron coincide with those of the four-dimensional basic simplex, $\psi_{j l}$ (cf. eqs. (2.24), (2.27)). The dual matrix $\left\|\widetilde{c}_{j l}\right\|$ as well as its determinant $\widetilde{D}^{(4)}$, cf. eq. (2.26),

$$
\left\|\widetilde{c}_{j l}\right\|=\left(\begin{array}{cccc}
1 & \widetilde{c}_{12} & \widetilde{c}_{13} & \widetilde{c}_{14} \\
\widetilde{c}_{12} & 1 & \widetilde{c}_{23} & \widetilde{c}_{24} \\
\widetilde{c}_{13} & \widetilde{c}_{23} & 1 & \widetilde{c}_{34} \\
\widetilde{c}_{14} & \widetilde{c}_{24} & \widetilde{c}_{34} & 1
\end{array}\right), \quad \widetilde{c}_{j l}=-\cos \psi_{j l}, \quad \widetilde{D}^{(4)}=\operatorname{det}\left\|\widetilde{c}_{j l}\right\|,
$$

are usually referred to as the Gram matrix and determinant of the spherical or hyperbolic tetrahedron. For example, the dihedral angle at the edge 12 of the spherical tetrahedron

\footnotetext{
${ }^{14}$ In the hyperbolic case, this is a hyperbolic tetrahedron whose volume can be obtained by analytic continuation (see below).

${ }^{15}$ It should be stressed that Fig. 10 is just an illustration (rather than precise picture) since a realistic non-Euclidean tetrahedron should be understood as embedded into four-dimensional Euclidean space.
} 
in Fig. 10 is $\psi_{34}$, etc. hyperbolic spaces, i.e. it remains unchanged along the given edge.

Unfortunately, there are no simple relations like (5.4) which might make it possible to express the volume of a spherical (or hyperbolic) tetrahedron in terms of its sides or dihedral angles. In fact, calculation of this volume in an elliptic or hyperbolic space is a well-known problem of non-Euclidean geometry (see e.g. in [36, 37, 38]). A standard way to solve this problem, say in spherical space, is to split an arbitrary tetrahedron into a set of birectangular ones. For example, the tetrahedron 1234 (shown in Fig. 10) is called birectangular (or double-rectangular) if (i) the edge 12 is perpendicular to the face 234 and (ii) the opposite edge, 34, is perpendicular to the face 123. It is easy to check that in this case three dihedral angles, $\psi_{13}, \quad \psi_{14}$ and $\psi_{24}$, are right angles. The other three are usually denoted [39, 40] as

$$
\psi_{12}=\alpha, \quad \psi_{23}=\beta, \quad \psi_{34}=\gamma
$$

The volume of a birectangular tetrahedron is known (see below) and can be expressed in terms of Lobachevsky or Schläfli functions which can be related to dilogarithms (see in [39, 40]).

The volume of the general tetrahedron must be a symmetrical function of the six edges $\tau_{j l}$, or equivalently of the six dihedral angles $\psi_{j l}$. When we break it up into a sum of birectangular tetrahedra, this explicit symmetry may be lost, although of course it must be hidden in the properties of the sums of dilogarithmic functions.

The general way to split an arbitrary tetrahedron into a sum of birectangular ones is to fix a point 0 , say inside the tetrahedron $\square$. Then, connecting this point 0 with each of the vertices of the tetrahedron 1234, we split it into four smaller tetrahedra: 0123, 0124, 0134 and 0234. Consider one of these tetrahedra, say 0123. Dropping the perpendicular from 0 onto the face 123 and connecting the foot of this perpendicular $F_{123}$ with the vertices 1,2 and 3 , we split 0123 into three rectangular tetrahedra, $012 F_{123}, 013 F_{123}$ and $023 F_{123}$. Then, each of these rectangular tetrahedra (say, $012 F_{123}$ ) can be split into a sum of two birectangular tetrahedra by dropping a perpendicular from $F_{123}$ onto the only side belonging to the original tetrahedron 1234 (the edge 12). In this way, we get $4 \times 3 \times 2=24$ birectangular tetrahedra.

Since we are free to choose the position of the point 0 , there are (at least) two ways to reduce the number of terms involved in this volume splitting in the general case:

(i) The point 0 may coincide with one of the vertices of the original tetrahedron, say with the vertex 4 (cf. Fig. 11a). In this case, we do not need to split into four smaller tetrahedra and can start just by dropping a perpendicular from the point 4 onto the face 123. In this way, we reduce the number of birectangular tetrahedra involved to six. However, the price we pay for this is the loss of explicit symmetry.

(ii) A symmetric choice of the point 0 can be related to the structure of the basic fourdimensional Euclidean simplex. In particular, we can take as the point 0 the intersection of the height $H_{0}$ and the unit hypersphere. Moreover, since the general four-point function

\footnotetext{
${ }^{16}$ The digits labelling the edge and the dihedral angle at this edge should cover the whole set 1234 .

${ }^{17}$ If this point (or the feet of some of the perpendiculars) happen to be outside the tetrahedron, this would mean that some of the volumes of the resulting birectangular tetrahedra should be taken with an opposite sign.
} 
can be reduced to the equal-mass case (see eqs. (3.10)-(3.11)), it is enough to consider the case when the point 0 is equidistant from the vertices $1,2,3$ and 4 . In this case, at the first stage of splitting we get four "isosceles" tetrahedra, signifying for each of them the three sides meeting at the point 0 are equal. Continuing the splitting, we see that due to "isoscelesness" the pairs of the tetrahedra produced at the last stage have equal values. Effectively, we get the sum of $4 \times 3=12$ volumes of birectangular tetrahedra. Although this is twice as much as in the case (i), the advantage is that we are able to preserve the explicit symmetry at all stages.

We note that the possibility to rescale the Feynman parameters 5 in the case $n=N$ is closely connected with the freedom to choose the point 0 as we like. As we have seen, this rescaling preserves the angles $\tau_{j l}$ between the mass vectors, whereas the direction of the height $H_{0}$ varies. In particular, we can make it coincide with with one of the mass vectors, which corresponds to the splitting (i) considered above. From this point of view, we lose the explicit symmetry since the corresponding rescaling is not symmetric.

Another possibility to reduce the number of birectangular volumes involved is to try to choose the point 0 in such a way that the volumes of the four tetrahedra (produced at the first stage of splitting) are equal (or proportional) to each other. In this case, the further splitting would give just $3 \times 2=6$ birectangular terms, i.e. the same number as in the case (i); but here we may preserve the explicit symmetry. However, it seems to be difficult (if possible at all) to define the position of the point 0 in this case, because one needs to solve a set of transcendental equations.

In some special examples described below we show that an additional symmetry of the diagram can also be employed.

\subsection{Volume of birectangular tetrahedra in curved space}

As we have seen (cf. eq. (6.5)), the birectangular tetrahedron may be specified by three dihedral angles $\psi_{12}=\alpha, \psi_{23}=\beta, \psi_{34}=\gamma$, so that the corresponding Gram matrix $\left\|\widetilde{c}_{j l}\right\|$ and its determinant (cf. eq. (20) of [40]) are

$$
\left\|\widetilde{c}_{j l}\right\|=\left(\begin{array}{cccc}
1 & -\cos \alpha & 0 & 0 \\
-\cos \alpha & 1 & -\cos \beta & 0 \\
0 & -\cos \beta & 1 & -\cos \gamma \\
0 & 0 & -\cos \gamma & 1
\end{array}\right), \quad \widetilde{D}^{(4)}=\sin ^{2} \alpha \sin ^{2} \gamma-\cos ^{2} \beta
$$

The volume of this birectangular tetrahedron (i.e. its contribution to $\Omega^{(4)}$ ) can be presented as

$$
\Omega^{(4)}=V(\alpha, \beta, \gamma)=\frac{1}{4} S(\pi / 2-\alpha, \beta, \pi / 2-\gamma)
$$

The notation $S(\alpha, \beta, \gamma)$ corresponds to the Schläfli function as defined in [39], whereas the notation $V(\alpha, \beta, \gamma)$ was used in [40] and is proportional to the function originally used by Schläfli [37]. Note that the function (6.7) is symmetric with respect to $\alpha$ and $\gamma$.

\footnotetext{
${ }^{18}$ Cf. eqs. (3.27)-(3.28): for $n=N$, the denominator disappears. In general, the $f_{i}$ can take arbitrary positive values.
} 
If we define (cf. eq. (3.21) of [39])

$$
X \equiv \frac{\cos \alpha \cos \gamma-\sqrt{\widetilde{D}^{(4)}}}{\cos \alpha \cos \gamma+\sqrt{\widetilde{D}^{(4)}}}
$$

the result for the volume can be expressed in terms of dilogarithms [39],

$$
\begin{aligned}
& 4 V(\alpha, \beta, \gamma)=\frac{1}{2}\left[\operatorname{Li}_{2}\left(X e^{2 i \alpha}\right)+\operatorname{Li}_{2}\left(X e^{-2 i \alpha}\right)\right]+\frac{1}{2}\left[\operatorname{Li}_{2}\left(X e^{2 i \gamma}\right)+\operatorname{Li}_{2}\left(X e^{-2 i \gamma}\right)\right] \\
& -\frac{1}{2}\left[\operatorname{Li}_{2}\left(-X e^{2 i \beta}\right)+\operatorname{Li}_{2}\left(-X e^{-2 i \beta}\right)\right]-\operatorname{Li}_{2}(-X)-\left(\frac{\pi}{2}-\alpha\right)^{2}-\left(\frac{\pi}{2}-\gamma\right)^{2}+\beta^{2} .(6
\end{aligned}
$$

When the condition $\widetilde{D}^{(4)} \geq 0(\sin \alpha \sin \gamma \leq \cos \beta)$ is obeyed, the tetrahedron exists in the usual geometrical sense, and its volume is real. In this case, one can express $V(\alpha, \beta, \gamma)$ in terms of the real function $\operatorname{Li}_{2}(r, \theta) \equiv \operatorname{Re}\left[\operatorname{Li}_{2}\left(r e^{i \theta}\right)\right]$ (see in Appendix A). However, one should distinguish between the cases $X \geq 0\left(\right.$ or $\left.\cos ^{2} \alpha+\cos ^{2} \beta+\cos ^{2} \gamma \geq 1\right)$ and $X \leq 0$ (or $\cos ^{2} \alpha+\cos ^{2} \beta+\cos ^{2} \gamma \leq 1$ ). The corresponding expressions are

$$
\begin{gathered}
\left.4 V(\alpha, \beta, \gamma)\right|_{X \geq 0}=\operatorname{Li}_{2}(X, 2 \alpha)+\operatorname{Li}_{2}(X, 2 \gamma)-\operatorname{Li}_{2}(X, \pi-2 \beta)-\operatorname{Li}_{2}(-X) \\
-\left(\frac{\pi}{2}-\alpha\right)^{2}-\left(\frac{\pi}{2}-\gamma\right)^{2}+\beta^{2}, \\
\begin{aligned}
\left.4 V(\alpha, \beta, \gamma)\right|_{X \leq 0}=\operatorname{Li}_{2}(-X, \pi-2 \alpha)+\operatorname{Li}_{2}(-X, \pi-2 \gamma)-\operatorname{Li}_{2}(-X, 2 \beta)-\operatorname{Li}_{2}(-X) \\
-\left(\frac{\pi}{2}-\alpha\right)^{2}-\left(\frac{\pi}{2}-\gamma\right)^{2}+\beta^{2} .
\end{aligned}
\end{gathered}
$$

In particular, when $X=-1$ we take into account that $\operatorname{Li}_{2}(1, \theta)=\frac{1}{4}(\pi-\theta)^{2}-\frac{1}{12} \pi^{2}$ and get

$$
\left.V(\alpha, \beta, \gamma)\right|_{X=-1}=\frac{1}{4} \pi(\alpha+\beta+\gamma-\pi) .
$$

When $\alpha=\beta=\gamma=\frac{1}{2} \pi$, this gives $\frac{1}{8} \pi^{2}$, which agrees with eq. (3.14) (at $N=4, \nu_{i}=1$ ).

When $\widetilde{D}^{(4)}<0 \quad(\sin \alpha \sin \gamma<\cos \beta)$, the tetrahedron does not exist in the usual geometrical sense (in the spherical case), but its volume (more precisely, the analytic continuation of the function corresponding to the volume) can be obtained from eq. (6.9), and it is imaginary. In this case we define (according to refs. [39, 40])

$$
X=e^{-2 i \delta}, \quad \text { so that } \quad \tan \delta=\frac{\sqrt{-\widetilde{D}^{(4)}}}{\cos \alpha \cos \gamma}
$$

and we get

$$
\begin{array}{r}
V(\alpha, \beta, \gamma)=\frac{1}{8 \mathrm{i}}\left[\mathrm{Cl}_{2}(2 \alpha+2 \delta)-\mathrm{Cl}_{2}(2 \alpha-2 \delta)+\mathrm{Cl}_{2}(2 \gamma+2 \delta)-\mathrm{Cl}_{2}(2 \gamma-2 \delta)\right. \\
\left.-\mathrm{Cl}_{2}(\pi-2 \beta+2 \delta)+\mathrm{Cl}_{2}(\pi-2 \beta-2 \delta)+2 \mathrm{Cl}_{2}(\pi-2 \delta)\right]
\end{array}
$$

Using the connection between the Clausen function and the Lobachevsky function $L(\theta)$ (cf. eq. (A.10) in Appendix A), eq. (6.14) can be re-written in terms of Lobachevsky function $[9$. The same expression (6.14) gives the volume of a birectangular tetrahedron in the hyperbolic space (see in [39, 40]).

\footnotetext{
${ }^{19}$ The function used in [40] which was denoted by a Cyrillic "L" can be expressed in terms of the Clausen function as $\frac{1}{2} \mathrm{Cl}_{2}(2 \theta)$.
} 


\subsection{Some cases with an additional symmetry}

There are a few cases of particular practical interest because of their inherent higher symmetries. In particular, there is the most symmetrical case where all $c_{i j}=c$. Thus all dihedral angles of the full (regular) tetrahedron are equal, to $\psi$ say. In that case the six birectangular tetrahedra corresponding to splitting (i) are all of equal size and $\Omega^{(4)}$ reduces to

$$
\Omega^{(4)}=6 V(\pi / 3, \psi / 2, \psi)=\frac{3}{2} S(\pi / 6, \psi / 2, \pi / 2-\psi) .
$$

Coxeter gives the identities (eqs. (4.31)-(4.32) of [39])

$$
S(\pi / 6, \psi / 2, \pi / 2-\psi)=4 S(\pi / 6, \pi / 3,(\pi-\psi) / 2)=\frac{2}{3} S((\pi-\psi) / 2, \psi,(\pi-\psi) / 2),
$$

which he derived algebraically, and which can be used to convert $\Omega^{(4)}$ given by eq. (6.7) into other forms. We will presently explain the geometrical meaning of these identities.

Another case of interest corresponds to equal mass scattering with equal internal masses: this means $c_{12}=c_{23}=c_{34}=c_{14}$ and in general are not equal to $c_{13}, c_{24}$, which are associated with energy and scattering angle variables. This case has four of the tetrahedral sides equal and different from the other two (which are opposite to one another). Thus there are three distinct $c$ 's. We shall see that it is possible here to break up full tetrahedron into four birectangular ones by joining the midpoints of the unequal sides to render the problem more tractable and make the maximum use of what symmetry exists.

We shall carry out our analysis by relaxing the symmetry relations. First we consider the case where all six $c_{i j}$ are equal to $c$ and treat this in three different ways. Then we let $c_{13} \equiv c_{s}$ and $c_{24} \equiv c_{t}$ be unequal.

Completely symmetrical box diagram. Here all $c_{j l}$ are equal and we are dealing with a completely regular tetrahedron. For example, in the equal-mass case this would mean that all $k_{j l}^{2}$ are equal巴ण, to $k^{2}$ say, and all $c=1-k^{2} /\left(2 m^{2}\right)$. We have

$$
\left\|c_{j l}\right\|=\left(\begin{array}{cccc}
1 & c & c & c \\
c & 1 & c & c \\
c & c & 1 & c \\
c & c & c & 1
\end{array}\right), \quad\left\|\widetilde{c}_{j l}\right\|=\left(\begin{array}{cccc}
1 & \widetilde{c} & \widetilde{c} & \widetilde{c} \\
\widetilde{c} & 1 & \widetilde{c} & \widetilde{c} \\
\widetilde{c} & \widetilde{c} & 1 & \widetilde{c} \\
\widetilde{c} & \widetilde{c} & \widetilde{c} & 1
\end{array}\right), \quad \widetilde{c}=-\frac{c}{1+2 c} .
$$

This means that the dihedral angle $\psi$ between each and every pair of hyperplanes ${ }^{2}$ equals $\arccos \left(\frac{c}{1+2 c}\right)$.

We can evaluate the hypervolume in at least three ways (the first two correspond to the ways (i) and (ii) considered in the general case):

(i) By dropping a perpendicular from one corner onto the opposite hyperplane and another perpendicular onto one of the opposite sides, we subdivide the full tetrahedron into six equal birectangular tetrahedra (see Fig. 11a). By inspection, each birectangular tetrahedron has dihedral angles $\psi, \psi / 2$ and $\pi / 3$. Therefore the total hypervolume equals

$$
\Omega^{(4)}=6 V(\psi, \psi / 2, \pi / 3)=\frac{3}{2} S(\pi / 6, \psi / 2, \pi / 2-\psi) .
$$

\footnotetext{
${ }^{20}$ Of course this represents an unphysical point in the Mandelstam diagram.

${ }^{21}$ The generalization of this totally symmetric case to an $N$-point function is quite easy and leads to $\cos \psi=c /[1+(N-2) c]$.
} 
(ii) We can alternatively consider the total volume as four times the volume contained in the tetrahedron generated by one base triangle and the "centre of mass" point 0 : $P_{0}=\frac{1}{4}\left(P_{1}+P_{2}+P_{3}+P_{4}\right)$ (here, we use the notation $P_{i}=m_{i} a_{i}$, cf. eq. (2.49)). In this case the matrix associated with the tetrahedron 0123 is found to be

$$
\left\|c_{j l}\right\|=\left(\begin{array}{cccc}
1 & c^{\prime} & c^{\prime} & c^{\prime} \\
c^{\prime} & 1 & c & c \\
c^{\prime} & c & 1 & c \\
c^{\prime} & c & c & 1
\end{array}\right), \quad c^{\prime}=\frac{\sqrt{1+3 c}}{2}
$$

leading to

$$
\left\|\widetilde{c}_{j l}\right\|=\left(\begin{array}{cccc}
1 & \widetilde{c}^{\prime} & \widetilde{c}^{\prime} & \widetilde{c}^{\prime} \\
\widetilde{c}^{\prime} & 1 & 1 / 2 & 1 / 2 \\
\widetilde{c}^{\prime} & 1 / 2 & 1 & 1 / 2 \\
\widetilde{c}^{\prime} & 1 / 2 & 1 / 2 & 1
\end{array}\right), \quad \widetilde{c}^{\prime}=-\sqrt{\frac{1+3 c}{2(1+2 c)}} .
$$

This implies that there are two distinct dihedral angles,

$$
\frac{2}{3} \pi \quad \text { and } \quad \arccos \sqrt{\frac{1+3 c}{2(1+2 c)}}=\frac{1}{2} \arccos \left(\frac{c}{1+2 c}\right)=\frac{\psi}{2}
$$

in fact. Actually the result is hardly surprising since it can be deduced by simple inspection of the geometrical figure. Anyhow if we now divide 0123 into six identical birectangular tetrahedra by dropping a perpendicular from 0 onto the centre of the triangle 123, these smaller tetrahedra possess dihedral angles $\psi / 2, \pi / 3, \pi / 3$. Consequently we have an alternative geometrical expression for the full volume,

$$
\Omega^{(4)}=24 V(\pi / 3, \pi / 3, \psi / 2)=6 S(\pi / 6, \pi / 3,(\pi-\psi) / 2) .
$$

Comparing this with the previous answer we have a geometrical explanation of one of the Coxeter identities (6.16). Furthermore, because (see [39], p. 18) there exists the differential relation

$$
\mathrm{d} S(\pi / 6, \pi / 3, \gamma)=-2 \arccos \left(\frac{\cos \gamma}{\sqrt{4 \cos ^{2} \gamma-1}}\right) \mathrm{d} \gamma
$$

we get the following integral representation of the four-dimensional solid angle (6.22):

$$
\Omega^{(4)}=\pi^{2}-12 \int_{0}^{(\pi-\psi) / 2} \mathrm{~d} \gamma \arccos \left(\frac{\cos \gamma}{\sqrt{4 \cos ^{2} \gamma-1}}\right) .
$$

(iii) We take the two midpoints $S$ and $T$ of the opposite sides: $P_{S}=\left(P_{1}+P_{3}\right) / 2, \quad P_{T}=$ $\left(P_{2}+P_{4}\right) / 2$ (see in Fig. 11b). The full volume is then just four times that of the tetrahedron $12 S T$, which is already birectangular as can be seen by inspection. In this case the matrix $\left\|c_{j l}\right\|$ associated with the tetrahedron $12 S T$ has the following non-diagonal elements:

$$
c_{12}=c, \quad c_{13}=c_{24}=\sqrt{\frac{1+c}{2}}, \quad c_{14}=c_{23}=c \sqrt{\frac{2}{1+c}}, \quad c_{34}=\frac{2 c}{1+c} .
$$


The corresponding Gram matrix of the tetrahedron 12ST is

$$
\left\|\widetilde{c}_{j l}\right\|=\left(\begin{array}{cccc}
1 & 0 & \widetilde{c}^{\prime} & 0 \\
0 & 1 & 0 & \widetilde{c}^{\prime} \\
\widetilde{c}^{\prime} & 0 & 1 & \widetilde{c} \\
0 & \widetilde{c}^{\prime} & \widetilde{c} & 1
\end{array}\right),
$$

with $\widetilde{c}$ and $\widetilde{c}^{\prime}$ defined in eqs. (6.17) and (6.20), respectively. From this we extract the three dihedral angles,

$$
\alpha=\gamma=\frac{1}{2} \psi \quad \text { and } \quad \beta=\psi,
$$

where $\psi$ is nothing but the dihedral angle at the side 12 , i.e. one of the dihedral angles of the full tetrahedron. This accords completely with the shape of $12 S T$. Hence the total volume of the complete tetrahedron 1234 is given by

$$
\Omega^{(4)}=4 V(\psi / 2, \psi, \psi / 2)=S((\pi-\psi) / 2, \psi,(\pi-\psi) / 2) .
$$

Comparing with the earlier expression, this provides a geometric meaning of the second Coxeter identity (6.16).

Partially symmetric box diagram. In this case we shall take four sides of the tetrahedron to be equal and two opposite sides to be unequal to them. In the equal-mass case, this would correspond to setting $k_{12}^{2}=k_{23}^{2}=k_{34}^{2}=k_{14}^{2}=k^{2}, k_{13}^{2}=s, k_{24}^{2}=t$, and we would get three different $c^{\prime}$ s: $c=1-k^{2} / 2 m^{2}, c_{s}=1-s / 2 m^{2}, c_{t}=1-t / 2 m^{2}$ ( $s$ and $t$ are just the Mandelstam variables). In this case much the simplest way of evaluating the volume of the tetrahedron is to break it up into four equal birectangular tetrahedra by taking the midpoints of the sides 13 and 24, as before: $P_{S}=\left(P_{1}+P_{3}\right) / 2, P_{T}=\left(P_{2}+P_{4}\right) / 2$. There is enough symmetry in the problem that the full tetrahedron is four times the birectangular tetrahedron $12 S T$. The latter is described by the matrix $\left\|c_{j l}\right\|$ with the non-diagonal elements

$$
\begin{gathered}
c_{12}=c, \quad c_{13}=\sqrt{\frac{1+c_{s}}{2}}, \quad c_{24}=\sqrt{\frac{1+c_{t}}{2}} \\
c_{23}=c \sqrt{\frac{2}{1+c_{s}}}, \quad c_{14}=c \sqrt{\frac{2}{1+c_{t}}}, \quad c_{34}=\frac{2 c}{\sqrt{\left(1+c_{s}\right)\left(1+c_{t}\right)}} .
\end{gathered}
$$

The non-diagonal elements of the "dual" matrix $\left\|\widetilde{c}_{j l}\right\|$ are

$$
\begin{gathered}
\widetilde{c}_{13}=-\sqrt{\frac{\left(1+c_{s}\right)\left(1+c_{t}\right)-4 c^{2}}{2\left(1+c_{t}-2 c^{2}\right)}}, \quad \widetilde{c}_{24}=-\sqrt{\frac{\left(1+c_{s}\right)\left(1+c_{t}\right)-4 c^{2}}{2\left(1+c_{s}-2 c^{2}\right)}}, \\
\widetilde{c}_{34}=-c \sqrt{\frac{\left(1-c_{s}\right)\left(1-c_{t}\right)}{\left(1+c_{s}-2 c^{2}\right)\left(1+c_{t}-2 c^{2}\right)}}, \quad \widetilde{c}_{12}=\widetilde{c}_{14}=\widetilde{c}_{23}=0 .
\end{gathered}
$$

We can now read off the three non-trivial dihedral angles,

$$
\begin{gathered}
\alpha=\arccos \left(\sqrt{\frac{\left(1+c_{s}\right)\left(1+c_{t}\right)-4 c^{2}}{2\left(1+c_{t}-2 c^{2}\right)}}\right), \quad \gamma=\arccos \left(\sqrt{\frac{\left(1+c_{s}\right)\left(1+c_{t}\right)-4 c^{2}}{2\left(1+c_{s}-2 c^{2}\right)}}\right), \\
\beta=\arccos \left(c \sqrt{\frac{\left(1-c_{s}\right)\left(1-c_{t}\right)}{\left(1+c_{s}-2 c^{2}\right)\left(1+c_{t}-2 c^{2}\right)}}\right) .
\end{gathered}
$$


Finally then the required volume of the full tetrahedron 1234 is obtained from

$$
\Omega^{(4)}=4 V(\alpha, \beta, \gamma)=S(\pi / 2-\alpha, \beta, \pi / 2-\gamma)
$$

A well-known physical example of partially symmetric box diagram is related to the photon-photon scattering [41]. In this case, $k^{2}=0, c=1, \cos \beta=1, \beta=0$. Furthermore, $\widetilde{D}^{(4)}=\sin ^{2} \alpha \sin ^{2} \gamma-1$ and we should use the formula 6.14) for $V(\alpha, 0, \gamma)$.

\subsection{The massless limit}

In the case when all masses $m_{i}$ vanish, the quantities $c_{j l}$ become infinite (cf. eq. (2.4)) and should be be considered as hyperbolic cosines. The determinant $D^{(4)}$, eq. (6.2), has the following limit:

$$
\begin{gathered}
\left.\left(m_{1}^{2} m_{2}^{2} m_{3}^{2} m_{4}^{2} D^{(4)}\right)\right|_{m_{i} \rightarrow 0} \\
\Rightarrow \frac{1}{16}\left[\left(k_{12}^{2} k_{34}^{2}\right)^{2}+\left(k_{13}^{2} k_{24}^{2}\right)^{2}+\left(k_{14}^{2} k_{23}^{2}\right)^{2}-2 k_{12}^{2} k_{34}^{2} k_{13}^{2} k_{24}^{2}-2 k_{12}^{2} k_{34}^{2} k_{14}^{2} k_{23}^{2}-2 k_{13}^{2} k_{24}^{2} k_{14}^{2} k_{23}^{2}\right] \\
=\frac{1}{16} \lambda\left(k_{12}^{2} k_{34}^{2}, k_{13}^{2} k_{24}^{2}, k_{14}^{2} k_{23}^{2}\right)
\end{gathered}
$$

with $\lambda(x, y, z)$ defined by eq. (1.1).

The elements of the dual matrix $\left\|\widetilde{c}_{j l}\right\|$ still can be interpreted as cosines of the dihedral angles, namely:

$$
\begin{aligned}
& \widetilde{c}_{12}=\widetilde{c}_{34}=-\cos \psi_{34}=-\cos \psi_{12}=\frac{k_{13}^{2} k_{24}^{2}+k_{14}^{2} k_{23}^{2}-k_{12}^{2} k_{34}^{2}}{\sqrt{k_{13}^{2} k_{24}^{2} k_{14}^{2} k_{23}^{2}}}, \\
& \widetilde{c}_{13}=\widetilde{c}_{24}=-\cos \psi_{24}=-\cos \psi_{13}=\frac{k_{14}^{2} k_{23}^{2}+k_{12}^{2} k_{34}^{2}-k_{13}^{2} k_{24}^{2}}{\sqrt{k_{14}^{2} k_{23}^{2} k_{12}^{2} k_{34}^{2}}}, \\
& \widetilde{c}_{14}=\widetilde{c}_{23}=-\cos \psi_{23}=-\cos \psi_{14}=\frac{k_{12}^{2} k_{34}^{2}+k_{13}^{2} k_{24}^{2}-k_{14}^{2} k_{23}^{2}}{\sqrt{k_{12}^{2} k_{34}^{2} k_{13}^{2} k_{24}^{2}}} .
\end{aligned}
$$

Therefore, in this situation we get nothing but an ideal hyperbolic tetrahedron (see pp. 39-40 of [40]), i.e. the tetrahedron whose vertices are all at infinity. The pairs of opposite dihedral angles of the ideal tetrahedron are equal, $\psi_{12}=\psi_{34}, \quad \psi_{13}=\psi_{24}, \quad \psi_{14}=$ $\psi_{23}$, whereas its volume is given by

$$
\Omega^{(4)}=\frac{1}{2 \mathrm{i}}\left[\mathrm{Cl}_{2}\left(2 \psi_{12}\right)+\mathrm{Cl}_{2}\left(2 \psi_{13}\right)+\mathrm{Cl}_{2}\left(2 \psi_{23}\right)\right], \quad \psi_{12}+\psi_{13}+\psi_{23}=\pi,
$$

according to eq. (41) of 40] (see also in 42]).

First of all we see that eqs. (6.33)-(6.36) depend only on the products $k_{12}^{2} k_{34}^{2}, k_{13}^{2} k_{24}^{2}$ and $k_{14}^{2} k_{23}^{2}$. This corresponds to the "glueing" of arguments in the massless case observed in ref. [43. Moreover, the result (6.37) is of the same form as the massless three-point function in four dimensions (1.2). This accords completely with the reduction of the massless four-point function to the three-point function which has been proven in [43]. 


\section{Reduction of integrals with $N>n$ and related problems}

\subsection{Integrals with $N>n$}

Consider eq. (3.45) in the case when $n=N-1$,

$$
J^{(N)}(N-1 ; 1, \ldots, 1)=\frac{1}{\Lambda^{(N)}}\left(\prod m_{i}^{2}\right) \sum_{i=1}^{N} \frac{1}{m_{i}^{2}} F_{i}^{(N)} J_{i}^{(N)}(N-1 ; 1, \ldots, 1) .
$$

The integrals on the r.h.s., $J_{i}^{(N)}(N-1 ; 1, \ldots, 1)$, correspond to splitting the basic $N$ dimensional simplex into $N$ rectangular ones. The $i$-th rectangular simplex is obtained via substituting the $i$-th mass side of the basic simplex by its height $H_{0}$ whose length is $m_{0}$. Therefore, the corresponding elements of the $\|c\|$ matrix (2.13) should be replaced by (cf. eqs. (3.43)-(3.44))

$$
c_{j i} \rightarrow \cos \tau_{0 j}=\frac{m_{0}}{m_{j}}, \quad c_{i l} \rightarrow \cos \tau_{0 l}=\frac{m_{0}}{m_{l}} .
$$

At this point let us consider what the representation (3.8) gives for $J_{i}^{(N)}$. The $\alpha$ integrand becomes

$$
\left(\sum_{l \neq i} \frac{\alpha_{l}}{m_{l}}+\frac{\alpha_{i}}{m_{0}}\right) \delta\left(\left.\left(\alpha^{T}\|c\| \alpha\right)\right|_{\alpha_{i}=0}+2 m_{0} \alpha_{i} \sum_{l \neq i} \frac{\alpha_{l}}{m_{l}}+\alpha_{i}^{2}-1\right) .
$$

One can see that the first factor in (7.3) is proportional to the derivative of the argument of the $\delta$ function with respect to $\alpha_{i}$. Therefore, eq. (7.3) can be expressed as

$$
\frac{1}{2 m_{0}} \frac{\partial}{\partial \alpha_{i}} \theta\left(\left.\left(\alpha^{T}\|c\| \alpha\right)\right|_{\alpha_{i}=0}+2 m_{0} \alpha_{i} \sum_{l \neq i} \frac{\alpha_{l}}{m_{l}}+\alpha_{i}^{2}-1\right) \text {. }
$$

Integrating over $\alpha_{i}$, we get

$$
J_{i}^{(N)}(N-1 ; 1, \ldots, 1)=\frac{\mathrm{i}^{1-2 N} \pi^{(N-1) / 2}}{m_{0}^{2} \prod_{l \neq i} m_{l}} \Gamma\left(\frac{N+1}{2}\right) \int_{0}^{\infty} \ldots \int_{0}^{\infty} \prod_{l \neq i} \mathrm{~d} \alpha_{l} \theta\left(1-\left.\left(\alpha^{T}\|c\| \alpha\right)\right|_{\alpha_{i}=0}\right) .
$$

Inserting

$$
\frac{1}{N-1} \sum_{l \neq i} \frac{\partial \alpha_{l}}{\partial \alpha_{l}}=1
$$

and integrating by parts, the integrand of (7.5) can be transformed as

$$
\begin{aligned}
\theta\left(1-\left.\left(\alpha^{T}\|c\| \alpha\right)\right|_{\alpha_{i}=0}\right) & \Rightarrow-\frac{1}{N-1}\left(\sum_{l \neq i} \alpha_{l} \frac{\partial}{\partial \alpha_{l}}\right) \theta\left(1-\left.\left(\alpha^{T}\|c\| \alpha\right)\right|_{\alpha_{i}=0}\right) \\
& \left.\Rightarrow \frac{1}{N-1} \delta\left(1-\left.\left(\alpha^{T}\|c\| \alpha\right)\right|_{\alpha_{i}=0}\right) \sum_{l \neq i} \alpha_{l} \frac{\partial}{\partial \alpha_{l}}\left(\alpha^{T}\|c\| \alpha\right)\right|_{\alpha_{i}=0} \\
& \Rightarrow \frac{2}{N-1} \delta\left(\left.\left(\alpha^{T}\|c\| \alpha\right)\right|_{\alpha_{i}=0}-1\right)
\end{aligned}
$$


Using eq. (3.8), we arrive at

$$
J_{i}^{(N)}(N-1 ; 1, \ldots, 1)=-\left.\frac{1}{2 m_{0}^{2}} J^{(N-1)}(N-1 ; 1, \ldots, 1)\right|_{\text {without } i} .
$$

Therefore, we get

$$
J^{(N)}(N-1 ; 1, \ldots, 1)=-\left.\frac{1}{2 D^{(N)}} \sum_{i=1}^{N} \frac{1}{m_{i}^{2}} F_{i}^{(N)} \quad J^{(N-1)}(N-1 ; 1, \ldots, 1)\right|_{\text {without } i} .
$$

This corresponds to eq. (47) of [11], when the number of external legs $N$ is equal to the space-time dimension plus one. For $N=3,4,5$, eq. (7.9) reproduces eqs. (10), (8), (13) from [1], respectively ${ }^{22}$.

Similar reduction formulae have also been considered in refs. [8]-[13]. In ref. [14] it was shown how the linear dependence of external momenta in the case $N \geq 6$ and for $n=4$ may be employed to reduce the $N$-point function to a set of $(N-1)$-point ones. A method for expressing the five-point function in four dimensions in terms of a sum of four-point functions was first shown in [7], whereas the reduction of the three-point function in two dimensions was studied in [10] (see also in [18]). These results were generalized in refs. [8, 9]. For example, in ref. [8] (see also in [10]) the representation for the coefficients corresponding to $F_{l}^{(N)}$ (eq. (7) of [8]) is given in terms of the propagators whose momenta are solutions to a certain set of equations corresponding to the other propagators. Moreover, it is possible to make a direct as opposed to step-by-step reduction to $n$-point functions for any $N>n$. More recently, some other approaches to this problem were suggested in [12, 13].

It is also instructive to consider another derivation of eq. (7.9). The representation (3.8) yields

$$
J^{(N)}(N-1 ; 1, \ldots, 1)=\frac{21^{1-2 N} \pi^{(N-1) / 2}}{\prod m_{l}} \Gamma\left(\frac{N+1}{2}\right) \int_{0}^{\infty} \ldots \int_{0}^{\infty} \prod \mathrm{d} \alpha_{l}\left(\sum_{i=1}^{N} \frac{\alpha_{i}}{m_{i}}\right) \delta\left(\alpha^{T}\|c\| \alpha-1\right) .
$$

Using eq. (2.33) we get

$$
\sum_{i=1}^{N} \frac{\alpha_{i}}{m_{i}}=\frac{1}{D^{(N)}} \sum_{i=1}^{N} \alpha_{i} \sum_{l=1}^{N} \frac{c_{i l}}{m_{l}} F_{l}^{(N)}=\frac{1}{D^{(N)}} \sum_{i=1}^{N} \frac{F_{i}^{(N)}}{m_{i}} \sum_{l=1}^{N} c_{i l} \alpha_{l} .
$$

Repeating the steps (7.3)-(7.7) for the resulting $\alpha$-integrals, we arrive at the following result:

$$
\begin{array}{r}
\frac{2 \mathrm{i}^{1-2 N} \pi^{(N-1) / 2}}{\prod m_{l}} \Gamma\left(\frac{N+1}{2}\right) \int_{0}^{\infty} \ldots \int_{0}^{\infty} \prod \mathrm{d} \alpha_{l}\left(\sum_{l=1}^{N} c_{i l} \alpha_{l}\right) \delta\left(\alpha^{T}\|c\| \alpha-1\right) \\
=-\left.\frac{1}{2 m_{i}} J^{(N-1)}(N-1 ; 1, \ldots, 1)\right|_{\text {without } i}
\end{array}
$$

Substituting (7.11) and (7.12) into (7.10), we obtain once again eq. (77.9).

Similar approach can also be used to obtain formulae connecting the integrals with different values of the space-time dimension [13, 44, 45].

\footnotetext{
${ }^{22}$ For $N=3$, the explicit result was presented in 10$]$.
} 


\subsection{Integrals with higher powers of propagators}

Equations like (7.12) can also be used for calculating integrals with higher powers of propagators. Such integrals can be associated with derivatives with respect to $m_{i}^{2}$, provided that $m_{i}$ and $k_{j l}^{2}$ are considered as the set of independent variables. Specifically, consider the sum[2]

$$
\begin{aligned}
& c_{1 i} m_{1} J^{(N)}(N+1 ; 2,1,1, \ldots, 1)+c_{2 i} m_{2} J^{(N)}(N+1 ; 1,2,1, \ldots, 1) \\
& \quad+\ldots+c_{i N} m_{N} J^{(N)}(N+1 ; 1, \ldots, 1,2) \equiv \sum_{l=1}^{N} c_{i l} m_{l} J^{(N)}\left(N+1 ;\left\{1+\delta_{l j}\right\}\right)
\end{aligned}
$$

(as usually, it is implied that $c_{j i}=c_{i j}$ and $c_{i i}=1$ ). Employing the representation (3.8) for all integrals in (7.13), we obtain the same integral as in eq. (7.12), multiplied by $(-\pi)$. Therefore, we get a system of equations which can be displayed in the matrix form,

$$
\left\|c_{j l}\right\|\left(\begin{array}{c}
m_{1} J^{(N)}(N+1 ; 2,1,1, \ldots, 1) \\
m_{2} J^{(N)}(N+1 ; 1,2,1, \ldots, 1) \\
\ldots \\
m_{N} J^{(N)}(N+1 ; 1,1, \ldots, 1,2)
\end{array}\right)=\frac{\pi}{2}\left(\begin{array}{c}
m_{1}^{-1} J^{(N-1)}(N-1 ; 1, \ldots, 1) \\
\left.m_{2}^{-1} J^{(N-1)}(N-1 ; 1, \ldots, 1)\right|_{\text {without 1 }} \\
\ldots \\
\left.m_{N}^{-1} J^{(N-1)}(N-1 ; 1, \ldots, 1)\right|_{\text {without } 2}
\end{array}\right) .
$$

The matrix inverse to $\left\|c_{j l}\right\|$ is given by eq. (2.25). Applying $\left\|c_{j l}\right\|^{-1}$ to both sides, we getter

$$
\begin{aligned}
& \left(\begin{array}{cc}
\left(m_{1} / \sqrt{D_{11}^{(N-1)}}\right) & J^{(N)}(N+1 ; 2,1,1, \ldots, 1) \\
\left.m_{2} / \sqrt{D_{22}^{(N-1)}}\right) & J^{(N)}(N+1 ; 1,2,1, \ldots, 1) \\
\left(m_{N} / \sqrt{D_{N N}^{(N-1)}}\right) & J^{(N)}(N+1 ; 1,1, \ldots, 1,2)
\end{array}\right) \\
& =\frac{\pi}{2 D^{(N)}}\left\|\widetilde{c}_{j l}\right\|\left(\begin{array}{cc}
m_{1}^{-1} \sqrt{D_{11}^{(N-1)}} & \left.J^{(N-1)}(N-1 ; 1, \ldots, 1)\right|_{\text {without 1 }} \\
m_{2}^{-1} \sqrt{D_{22}^{(N-1)}} & \left.J^{(N-1)}(N-1 ; 1, \ldots, 1)\right|_{\text {without 2 }} \\
m_{N}^{-1} \sqrt{D_{N N}^{(N-1)}} & \left.J^{(N-1)}(N-1 ; 1, \ldots, 1)\right|_{\text {without } N}
\end{array}\right)
\end{aligned}
$$

Using the expression (2.24) for $\widetilde{c}_{j N}$, we arrive at

$$
J^{(N)}\left(N+1 ;\left\{1+\delta_{i l}\right\}\right)=\left.\frac{\pi}{2 m_{l} D^{(N)}} \sum_{j=1}^{N} \frac{(-1)^{j+l}}{m_{j}} D_{j l}^{(N-1)} J^{(N-1)}(N-1 ; 1, \ldots, 1)\right|_{\text {without } j} .
$$

In particular, in the three-point four-dimensional case we end up with

$$
J^{(3)}(4 ; 1,1,2)=\frac{\mathrm{i} \pi^{2} \sin \tau_{12}}{2 m_{1} m_{2} m_{3}^{2} D^{(3)}}\left[\tau_{23} \cos \psi_{13}+\tau_{13} \cos \psi_{23}+\tau_{12}\right] .
$$

\footnotetext{
${ }^{23}$ The notation for the set of arguments $\left\{\nu_{j}\right\}=\left\{1+\delta_{l j}\right\}$ means that all powers of denominators are equal to one, except for the $\nu_{l}$ which is equal to two.

${ }^{24}$ Similar results can also be obtained using the integration-by-parts approach [46, 23].
} 
The results for $J^{(3)}(4 ; 1,2,1)$ and $J^{(3)}(4 ; 2,1,1)$ can be obtained by permutation of indices. The sum of these three integrals is in agreement with the result obtained in 18.

It is easy to check the consistency of the results (7.16) and (7.9). Using the standard Feynman parametric representation (or the representation (3.8) involving the $\delta$ function of the quadratic form) it is straightforward to show that

$$
\sum_{j=1}^{N} J^{(N)}\left(N+1 ;\left\{1+\delta_{i j}\right\}\right)=-\pi J^{(N)}(N-1 ; 1, \ldots, 1) .
$$

Considering the sum of the integrals (7.16) and employing the relation (7.18), we arrive at eq. (7.9).

\section{Conclusion}

Let us briefly summarize the main results of the present paper. We have proved that there is a direct link between Feynman parametric representation of a one-loop $N$-point function and the basic simplex in $N$-dimensional Euclidean space. To show this, we have changed the surface of integration in the Feynman parametric representation, from linear one $\left(\sum \alpha_{i}=1\right)$ to quadratic one $\left(\alpha^{T}\|c\| \alpha=1\right)$. The integral representation involving $\delta$ function of this quadratic form is given by eq. (3.8).

In the case $N=n$, the result for the Feynman integral turns out to be proportional to the ratio of an $N$-dimensional solid angle at the meeting point of the mass sides to the content of the $N$-dimensional basic simplex 20 . For example, to reproduce result for the three-dimensional three-point function [11] we just require the expression for the area of a spherical triangle which can easlily be calculated as the sum of the angles minus $\pi$ (in general, an analytic continuation of the area function should be taken).

For the four-dimensional four-point function, the representation (3.8) provides a very interesting connection with the volume of the non-Euclidean (spherical or hyperbolic) tetrahedron. By splitting the non-Euclidean tetrahedron into birectangular ones, the latter can be expressed in terms of dilogarithms or related functions ${ }^{27}$. Different ways of splitting correspond to different representations of the result for the four-point function. This "freedom" can be used to construct more compact representations (cf. refs. [3, 2, 四).

In other cases, such as for example the four-dimensional three-point function, we get an extra factor in the integrand corresponding to a power of the cosine of the angle between the height of the basic simplex and the vector of integration. In general, the height of the basic simplex, $H_{0}$, plays an essential role in calculation of the integrals with $N \neq n$. It is used to split the basic Euclidean simplex into $N$ rectangular simplices. When $N=n+1$, each integral corresponding to one of the resulting rectangular tetrahedra can be reduced to an $(N-1)$-point function. When $N<n$, this splitting also simplifies the calculation of separate integrals.

25 The generalization of this formula to the case of arbitrary $n$ and the powers of the propagators $\nu_{i}$ can be found e.g. in 477, eq. (6).

${ }^{26}$ Moreover, in the case $N=n$ the result for the diagram with unequal masses can be expressed in terms of the function corresponding to the equal-mass case.

${ }^{27} \mathrm{~A}$ compact integral representation for this volume can be found in ref. [48]. 
The derivations in this paper have been based on a purely geometrical approach to evaluation of $N$-point Feynman diagrams. In the resulting expressions, all arguments of functions arising possess a straightforward geometrical meaning in terms of the dihedral angles, etc. In particular, this is quite useful for choosing the most convenient kinematic variables to describe the $N$-point diagrams. We suggest that this approach can serve as a powerful tool for understanding the geometrical structure of loop integrals with several external legs, as well as the structure of the corresponding phase-space integrals (see e.g. in 49]). More optimistically, it may shed light on analytical results for higher loops.

Acknowledgements. One of the authors (A. D.) is grateful to the Australian Research Council for a grant which was used to support his visit to Hobart in 1996 (where an essential part of this work has been done), and to the Department of Physics, University

of Tasmania for hospitality during the visit. A. D.'s research was partly supported by the grants INTAS-93-0744 and RFBR-96-01-00654.

Note-added. After our manuscript has been accepted for a publication, the paper [50] appeared, dealing with non-relativistic Feynman integrals. It cited ref. [51] where the relativistic one-loop Feynman amplitudes (in Euclidean quantum field theory) were studied and expressed in terms of definite integrals over simplices. We also note that some representations for the Schläfli function (similar to those discussed in sec. 6.2) can be found in ref. [52].

\section{Appendix A: Dilogarithm and related functions}

Here we present definitions and some representations of the dilogarithm and related functions. More detailed information can be found in [27.

The Euler's dilogarithm is defined by

$$
\operatorname{Li}_{2}(z) \equiv-z \int_{0}^{1} \mathrm{~d} \xi \frac{\ln \xi}{1-\xi z}
$$

The Clausen function is related to the imaginary part of the dilogarithm,

$$
\mathrm{Cl}_{2}(\theta)=\operatorname{Im}\left[\operatorname{Li}_{2}\left(e^{\mathrm{i} \theta}\right)\right]=-\int_{0}^{\theta} \mathrm{d} \theta^{\prime} \ln \left|2 \sin \left(\frac{1}{2} \theta^{\prime}\right)\right|,
$$

and it can also be represented as

$$
\mathrm{Cl}_{2}(\theta)=-\sin \theta \int_{0}^{1} \frac{\mathrm{d} \xi \ln \xi}{1-2 \xi \cos \theta+\xi^{2}}
$$

Note that $\mathrm{Cl}_{2}(2 \pi-\theta)=-\mathrm{Cl}_{2}(\theta)$.

If we consider dilogarithm of a general complex argument, $\operatorname{Li}_{2}\left(r e^{\mathrm{i} \theta}\right)$, we get

$$
\operatorname{Re}\left[\operatorname{Li}_{2}\left(r e^{\mathrm{i} \theta}\right)\right] \equiv \operatorname{Li}_{2}(r, \theta)
$$




$$
\operatorname{Im}\left[\operatorname{Li}_{2}\left(r e^{\mathrm{i} \theta}\right)\right]=\omega \ln r+\frac{1}{2}\left[\mathrm{Cl}_{2}(2 \theta)+\mathrm{Cl}_{2}(2 \omega)+\mathrm{Cl}_{2}(2 \chi)\right],
$$

with $\tan \omega=r \sin \theta /(1-r \cos \theta)$ and $\chi \equiv \pi-\theta-\omega$. We note that

$$
\frac{1}{2}\left[\mathrm{Cl}_{2}(2 \theta)+\mathrm{Cl}_{2}(2 \omega)+\mathrm{Cl}_{2}(2 \chi)\right]=-\sin \theta \int_{0}^{r} \frac{\mathrm{d} \eta \ln \eta}{1-2 \eta \cos \theta+\eta^{2}}
$$

The imaginary part can also be rewritten as

$$
\operatorname{Im}\left[\operatorname{Li}_{2}\left(r e^{\mathrm{i} \theta}\right)\right]=\mathrm{Ti}_{2}(\tan \omega)-\mathrm{Ti}_{2}(\tan \omega, \tan \theta)=-r \sin \theta \int_{0}^{1} \frac{\mathrm{d} \xi \ln \xi}{1-2 r \xi \cos \theta+r^{2} \xi^{2}}
$$

where $\mathrm{Ti}_{2}(z)$ and $\mathrm{Ti}_{2}(z, a)$ are the ordinary and the generalized inverse tangent integrals, respectively:

$$
\mathrm{Ti}_{2}(z)=\int_{0}^{z} \frac{\mathrm{d} z^{\prime}}{z^{\prime}} \arctan z^{\prime}, \quad \operatorname{Ti}_{2}(z, a)=\int_{0}^{z} \frac{\mathrm{d} z^{\prime}}{z^{\prime}+a} \arctan z^{\prime} .
$$

In particular, $\mathrm{Ti}_{2}(z)=\mathrm{Ti}_{2}(z, 0)$.

Finally the Lobachevsky function $L(\theta)$ is defined by

$$
L(\theta) \equiv-\int_{0}^{\theta} \mathrm{d} \theta^{\prime} \ln \left(\cos \theta^{\prime}\right), \quad\left(-\frac{1}{2} \pi \leq \theta \leq \frac{1}{2} \pi\right)
$$

It is related to Clausen's function by

$$
\mathrm{Cl}_{2}(\theta)=-2 L\left(\frac{\pi-\theta}{2}\right)+(\pi-\theta) \ln 2, \quad L(\theta)=-\frac{1}{2} \mathrm{Cl}_{2}(\pi-2 \theta)+\theta \ln 2 .
$$

\section{Appendix B: On the rescaling of $\alpha$ 's}

Consider the transformation

$$
\alpha_{i}=\mathcal{G}\left(\alpha_{1}^{\prime}, \ldots, \alpha_{N}^{\prime}\right) \alpha_{i}^{\prime}, \quad i=1, \ldots, N
$$

where the "scaling" function $\mathcal{G}$ is the same for all $\alpha$ 's. The Jacobian of the transformation is

$$
\text { Jacobian }=\left(\prod_{j=1}^{N} \alpha_{j}^{\prime} \frac{\partial \mathcal{G}}{\partial \alpha_{j}^{\prime}}\right)\left|\begin{array}{cccc}
1+d_{1} & 1 & \ldots & 1 \\
1 & 1+d_{2} & \ldots & 1 \\
\ldots & \ldots & \ldots & \ldots \\
1 & 1 & \ldots & 1+d_{N}
\end{array}\right|, \quad d_{i} \equiv \mathcal{G}\left(\alpha_{i}^{\prime} \frac{\partial \mathcal{G}}{\partial \alpha_{i}^{\prime}}\right)^{-1} .
$$

Since the determinant in $(\mathrm{B} .2)$ is equal to

$$
\left(\prod_{i=1}^{N} d_{i}\right)\left[1+\frac{1}{d_{1}}+\frac{1}{d_{2}}+\ldots+\frac{1}{d_{N}}\right],
$$


the expression for the Jacobian reduces to

$$
\text { Jacobian }=\mathcal{G}^{N}\left(1+\frac{1}{\mathcal{G}} \sum_{j=1}^{N} \alpha_{j}^{\prime} \frac{\partial \mathcal{G}}{\partial \alpha_{j}^{\prime}}\right)=\mathcal{G}^{N}\left(1+\sum_{j=1}^{N} \alpha_{j}^{\prime} \frac{\partial(\ln \mathcal{G})}{\partial \alpha_{j}^{\prime}}\right)
$$

If $\mathcal{G}$ is a homogeneous function of $\alpha$ 's of the order $R$, i.e.

$$
\mathcal{G}\left(Z \alpha_{1}^{\prime}, \ldots, Z \alpha_{N}^{\prime}\right)=Z^{R} \mathcal{G}\left(\alpha_{1}^{\prime}, \ldots, \alpha_{N}^{\prime}\right)
$$

then

$$
\sum_{j=1}^{N} \alpha_{j}^{\prime} \frac{\partial \mathcal{G}}{\partial \alpha_{j}^{\prime}}=R \mathcal{G} \quad \text { and } \quad \text { Jacobian }=[1+R] \mathcal{G}^{N} .
$$

In particular, if $\mathcal{G}=Q_{r} / Q_{r^{\prime}}^{\prime}$, where $Q_{r}$ is a polyniomial of degree $r$ and $Q_{r^{\prime}}^{\prime}$ is a polynomial of degree $r^{\prime}$ in $\alpha^{\prime}$, Jacobian $\rightarrow\left[1+\left(r-r^{\prime}\right)\right] \mathcal{G}^{N}$, i.e. $R=r-r^{\prime}$. In the text we have encountered the situations when $r=r^{\prime}=1 \quad(R=0)$ and $r=2, r^{\prime}=1 \quad(R=1)$.

\section{References}

[1] L.M. Brown and R.P. Feynman, Phys. Rev. 85, 231 (1952);

G. Passarino and M. Veltman, Nucl. Phys. B160, 151 (1979).

[2] G. 'tHooft and M. Veltman, Nucl. Phys. B153, 365 (1979).

[3] A.C.T. Wu, Mat. Fys. Medd. Dan. Vid. Selsk. 33 (No.3), 1 (1961).

[4] A. Denner, U. Nierste and R. Scharf, Nucl. Phys. B367, 637 (1991).

[5] G.J. van Oldenborgh and J.A.M. Vermaseren, Z. Phys. C46, 425 (1990); G.J. van Oldenborgh, Phys. Lett. B282, 185 (1992).

[6] A. Denner, Fortschr. Phys. 41, 307 (1993).

[7] F.R. Halpern, Phys. Rev. Lett. 10, 310 (1963).

[8] B. Petersson, J. Math. Phys. 6, 1955 (1965).

[9] D.B. Melrose, Nuovo Cim. 40A, 181 (1965).

[10] G. Källén and J. Toll, J. Math. Phys. 6, 299 (1965).

[11] B.G. Nickel, J. Math. Phys. 19, 542 (1978).

[12] W.L. van Neerven and J.A.M. Vermaseren, Phys. Lett. B137, 241 (1984).

[13] Z. Bern, L. Dixon and D.A. Kosower, Phys. Lett. B302, 299 (1993); Nucl. Phys. B412, 751 (1994).

[14] L.M. Brown, Nuovo Cim. 22, 178 (1961). 
[15] D.S. Kershaw, J. Math. Phys. 15, 798 (1974); Phys. Rev. D8, 2708 (1973);

E.E. Boos and A.I. Davydychev, Theor. Math. Phys. 89, 1052 (1991);

D. Kreimer, Z. Phys. C54, 667 (1992); Int. J. Mod. Phys. A8, 1797 (1993);

L. Brücher, J. Franzkowski and D. Kreimer, Mod. Phys. Lett. A9, 2335 (1994).

[16] A.I. Davydychev, J. Math. Phys. 32, 1052 (1991); 33, 358 (1992).

[17] L.D. Landau, Nucl. Phys. 13, 181 (1959).

[18] G. Källen and A. Wightman, Mat. Fys. Skr. Dan. Vid. Selsk. 1 (No.6), 1 (1958).

[19] S. Mandelstam, Phys. Rev. 115, 1742 (1959);

R.E. Cutkosky, J. Math. Phys. 1, 429 (1960);

J.C. Taylor, Phys. Rev. 117, 261 (1960);

Yu.A. Simonov, Sov. Phys. JETP 16, 1599 (1963) [Zh. Eksp. Teor. Fiz. 43, 2263 (1962)].

[20] R.J. Eden, P.V. Landshoff, D.I. Olive and J.C. Polkinghorne, The analytic S-matrix (Cambridge UP, 1966).

[21] R. Karplus, C.M. Sommerfield and E.H. Wichmann, Phys. Rev. 114, 376 (1959).

[22] J.S. Ball and T.-W. Chiu, Phys. Rev. D22, 2550 (1980); D23, 3085(E) (1981);

H.J. Lu and C.A. Perez, preprint SLAC-PUB-5809, 1992.

[23] A.I. Davydychev, J. Phys. A25, 5587 (1992).

[24] J.J. van der Bij and M. Veltman, Nucl. Phys. B231, 205 (1984);

C. Ford, I. Jack and D.R.T. Jones, Nucl. Phys. B387, 373 (1992);

A.I. Davydychev and J.B. Tausk, Nucl. Phys. B397, 123 (1993).

[25] R. Scharf, Doctoral Thesis, Würzburg, 1994;

R. Scharf and J.B. Tausk, Nucl. Phys. B412, 523 (1994).

[26] A.I. Davydychev and J.B. Tausk, Phys. Rev. D53, 7381 (1996).

[27] L. Lewin, Polylogarithms and associated functions (North Holland, 1981).

[28] D.M.Y. Sommerville, An itroduction to the geometry of $N$ dimensions (Methuen, London, 1929).

[29] M.G. Kendall, A course in the geometry of $n$ dimensions (Charles Griffin, London, 1961).

[30] A.N. Vassiliev, Yu.M. Pis'mak and Yu.R. Honkonen, Teor. Mat. Fiz. 47, 291 (1981);

N.I. Ussyukina, Teor. Mat. Fiz. 54, 124 (1983);

D.I. Kazakov, Phys. Lett. B133, 406 (1983).

[31] A.K. Rajantie, Nucl. Phys. B480, 729 (1996). 
[32] G. 't Hooft and M. Veltman, Nucl. Phys. B44, 189 (1972);

C.G. Bollini and J.J. Giambiagi, Nuovo Cim. 12B, 20 (1972).

[33] F.A. Berends, A.I. Davydychev and V.A. Smirnov, Nucl. Phys. B478, 59 (1996).

[34] U. Nierste, D. Müller and M. Böhm, Z. Phys. C57, 605 (1993).

[35] E.E. Boos and A.I. Davydychev, Teor. Mat. Fiz. 89, 56 (1991).

[36] N.I. Lobatschefsky, Imaginäre Geometrie, Kasaner Gelehrte Schriften, 1836 (Übersetzung mit Anmerkungen von H. Liebmann, Leipzig, 1904).

[37] L. Schläfli, Quart. J. Math. 3, 54 (1860); 3, 97 (1860);

Gesammelte matematische Abhandlungen, Band II (Birkhäuser, Basel, 1953).

[38] J.L. Coolidge, The elements of non-Euclidean geometry (Clarendon Press, Oxford, 1909);

J. Böhm and E. Hertel, Polyedergeometrie in n-dimensionalen Räumen konstanter Krümmung (Birkhäuser, Basel, 1981).

[39] H.S.M. Coxeter, Quart. J. Math. 6, 13 (1935).

[40] E.B. Vinberg, Uspekhi Mat. Nauk 48 (No.2), 17 (1993) [Russian Math. Surveys 48 (No.2), 15 (1993)].

[41] R. Karplus and M. Neuman, Phys. Rev. 80, 380 (1950); 83, 776 (1951);

B. De Tollis, Nuovo Cim. 32, 757 (1964); 35, 1182 (1965).

[42] J. Milnor, Bull. Amer. Math. Soc. 6, 9 (1982).

[43] N.I. Ussyukina and A.I. Davydychev, Phys. Lett. B298, 363 (1993); B305, 136 (1993);

D.J. Broadhurst, Phys. Lett. B307, 132 (1993).

[44] Z. Bern and A.G. Morgan, Nucl. Phys. B467, 479 (1996).

[45] O.V. Tarasov, Phys. Rev. D54, 6479 (1996).

[46] F.V. Tkachov, Phys. Lett. B100, 65 (1981);

K.G. Chetyrkin and F.V. Tkachov, Nucl. Phys. B192, 159 (1981).

[47] A.I. Davydychev, Phys. Lett. B263, 107 (1991).

[48] W.-Y. Hsiang, Quart. J. Math. 39, 463 (1988).

[49] B. Almgren, Arkiv för Fysik 38 (No.7), 161 (1968);

E. Byckling and K. Kajantie, Particle kinematics (John Wiley, 1973).

[50] P. Wagner, J. Math. Phys. 39, 2428 (1998).

[51] N. Ortner and P. Wagner, Ann. Inst. Henri Poincaré (Phys. Théor.) 63, 81 (1995).

[52] R. Kellerhals, Math. Ann. 285, 541 (1989); in Structural Properties of Polylogarithms (ed. L. Lewin), AMS Math. Surveys and Monographs, vol. 37, p.301 (1991). 


\section{Figure captions}

Fig. 1: The triangle associated with the massless three-point function

Fig. 2: The one-loop $N$-point diagram

Fig. 3: The basic simplex for $N=4$

Fig. 4: Two-point case: (a) the basic triangle and (b) the arc $\tau_{12}$

Fig. 5: Three-point case: (a) the basic tetrahedron and (b) the solid angle

Fig. 6: The spherical triangle 123

Fig. 7: The isosceles spherical triangle 012

Fig. 8: Integration variables on the sphere

Fig. 9: An asymmetric spherical triangle 012

Fig. 10: The spherical tetrahedon 1234

Fig. 11: Different ways of splitting the non-Euclidean tetrahedron 